\title{
Facile Synthesis of Fused Oxasapphyrins
}

\author{
Prosenjit Isar and Mangalampalli Ravikanth* \\ Department of Chemistry, Indian Institute of Technology Bombay, Powai, Mumbai 400076, \\ India, E-mail: ravikanth@.chem.iitb.ac.in
}

\begin{tabular}{|c|c|c|}
\hline \multirow{4}{*}{\begin{tabular}{|l|} 
Scheme 1 \\
Scheme 2 \\
\end{tabular}} & Synthesis of napthodipyrrole-derived sapphyrin $\mathbf{1}$ & S2 \\
\hline & Synthesis of napthodipyrrole-derived oxasapphyrin 2 & S3 \\
\hline & Materials and methods & S4 \\
\hline & Synthesis & S6 \\
\hline Figure S1 & ${ }^{1} \mathrm{H}$ NMR of compound 5a recorded in $\mathrm{CDCl}_{3}$ & S11 \\
\hline Figure S2 & HRMS spectrum of compound $\mathbf{5 a}$ & S12 \\
\hline Figure S3 & ${ }^{13} \mathrm{NMR}$ of compound $\mathbf{5 a}$ recorded in $\mathrm{CDCl}_{3}$ & S13 \\
\hline Figure S4 & ${ }^{1} \mathrm{H}$ NMR of compound $\mathbf{5 b}$ recorded in $\mathrm{CDCl}_{3}$ & S14 \\
\hline Figure S5 & HRMS spectrum of compound $\mathbf{5 b}$ & S15 \\
\hline Figure S6 & ${ }^{13} \mathrm{NMR}$ of compound $\mathbf{5} \mathbf{b}$ recorded in $\mathrm{CDCl}_{3}$ & S16 \\
\hline Figure S7 & ${ }^{1} \mathrm{H}$ NMR of compound $\mathbf{6 a}$ recorded in $\mathrm{CDCl}_{3}$ & S17 \\
\hline Figure S8 & HRMS spectrum of compound $\mathbf{6 a}$ & S18 \\
\hline Figure S9 & ${ }^{13} \mathrm{NMR}$ of compound $\mathbf{6 a}$ recorded in $\mathrm{CDCl}_{3}$ & S19 \\
\hline Figure S10 & ${ }^{1} \mathrm{H}$ NMR of compound $\mathbf{6 b}$ recorded in $\mathrm{CDCl}_{3}$ & $\mathbf{S 2 0}$ \\
\hline Figure S11 & HRMS spectrum of compound $\mathbf{6 b}$ & S21 \\
\hline Figure S12 & ${ }^{13} \mathrm{NMR}$ of compound $\mathbf{6} \mathbf{b}$ recorded in $\mathrm{CDCl}_{3}$ & S22 \\
\hline Figure S13 & ${ }^{1} \mathrm{H}$ NMR of compound $\mathbf{6 c}$ recorded in $\mathrm{CDCl}_{3}$ & $\mathbf{S 2 3}$ \\
\hline Figure S14 & ${ }^{13} \mathrm{C}$ NMR of compound $\mathbf{6 c}$ recorded in $\mathrm{CDCl}_{3}$ & S24 \\
\hline Figure S15 & HRMS spectrum of compound $\mathbf{6 c}$ & S25 \\
\hline Figure S16 & ${ }^{1} \mathrm{H}$ NMR of compound 3a recorded in $\mathrm{CDCl}_{3}$ & S26 \\
\hline Figure S17 & HRMS spectrum of compound $\mathbf{3 a}$ & S27 \\
\hline Figure S18 & ${ }^{13} \mathrm{C}$ NMR of compound $\mathbf{3 a}$ recorded in $\mathrm{CDCl}_{3}$ & $\mathbf{S 2 8}$ \\
\hline Figure S19 & $\mathrm{D}_{2} \mathrm{O}$ exchange experiment in $\mathrm{CDCl}_{3}$ & $\mathbf{S 2 9}$ \\
\hline Figure S20 & Energy level diagram of compound $\mathbf{3 a}$ and $[\mathbf{3 a} . \mathbf{2 H}]^{2+}$. & S30 \\
\hline Figure S21 & NICS $(0)$ value of macrocycle $\mathbf{3 a}$ & S31 \\
\hline Figure S22 & ${ }^{1} \mathrm{H}$ NMR of compound $\mathbf{3 b}$ recorded in $\mathrm{CDCl}_{3}$ & S32 \\
\hline Figure S23 & HRMS spectrum of compound $\mathbf{3 b}$ & S33 \\
\hline Figure S24 & ${ }^{13} \mathrm{C}$ NMR of compound $\mathbf{3 b}$ recorded in $\mathrm{CDCl}_{3}$ & S34 \\
\hline Figure S25 & ${ }^{1} \mathrm{H}$ NMR of compound $\mathbf{3 c}$ recorded in $\mathrm{CDCl}_{3}$ & S35 \\
\hline Figure S26 & HRMS spectrum of compound $\mathbf{3 c}$ & S36 \\
\hline Figure S27 & ${ }^{13} \mathrm{C}$ NMR of compound $\mathbf{3 c}$ recorded in $\mathrm{CDCl}_{3}$ & S37 \\
\hline Figure S28 & $\begin{array}{l}\text { Table of absorption and electrochemical data of } \mathbf{3 a - 3 c} \text { along with } \\
\text { their diprotonated species }[\mathbf{3 a} . \mathbf{2 H}]^{2+}-[\mathbf{3 c} \cdot \mathbf{2} \mathbf{H}]^{2+}\end{array}$ & S38 \\
\hline Figure S29 & $\begin{array}{l}\text { Table of crystal data and data collection parameters for compound } \\
\text { 3a }\end{array}$ & S39 \\
\hline Figure S30 & Table of selected bond lengths $[\AA]$ and angles $\left[{ }^{\circ}\right]$ for compound $\mathbf{3 a}$ & S41 \\
\hline
\end{tabular}




\begin{tabular}{|l|l|c|}
\hline Figure S31 & Comparison of absorption spectra of 3a-3c and $[\mathbf{3 a . 2 H}]^{2+}-[\mathbf{3 c . 2 H}]^{2+}$ & $\mathbf{S 4 3}$ \\
\hline Figure S32 & Comparison of cyclic voltammogram of compounds 3a-3c & $\mathbf{S 4 3}$ \\
\hline Figure S33 & $\begin{array}{l}\text { Cartesian coordinates of the S0 optimized structures of the } \\
\text { compounds 3a }\end{array}$ & $\mathbf{S 4 4}$ \\
\hline Figure S34 & $\begin{array}{l}\text { Cartesian coordinates of the S0 optimized structures of the } \\
\text { compounds [3a.2H] }{ }^{++}\end{array}$ & $\mathbf{S 4 6}$ \\
\hline Figure S35 & $\begin{array}{l}\text { Normalized absorbance and fluorescence }\left(\lambda_{\text {ex }}=510 \mathrm{~nm}\right) \text { spectra of } \\
\text { compound 3a in chloroform }\end{array}$ & $\mathbf{S 4 7}$ \\
\hline
\end{tabular}

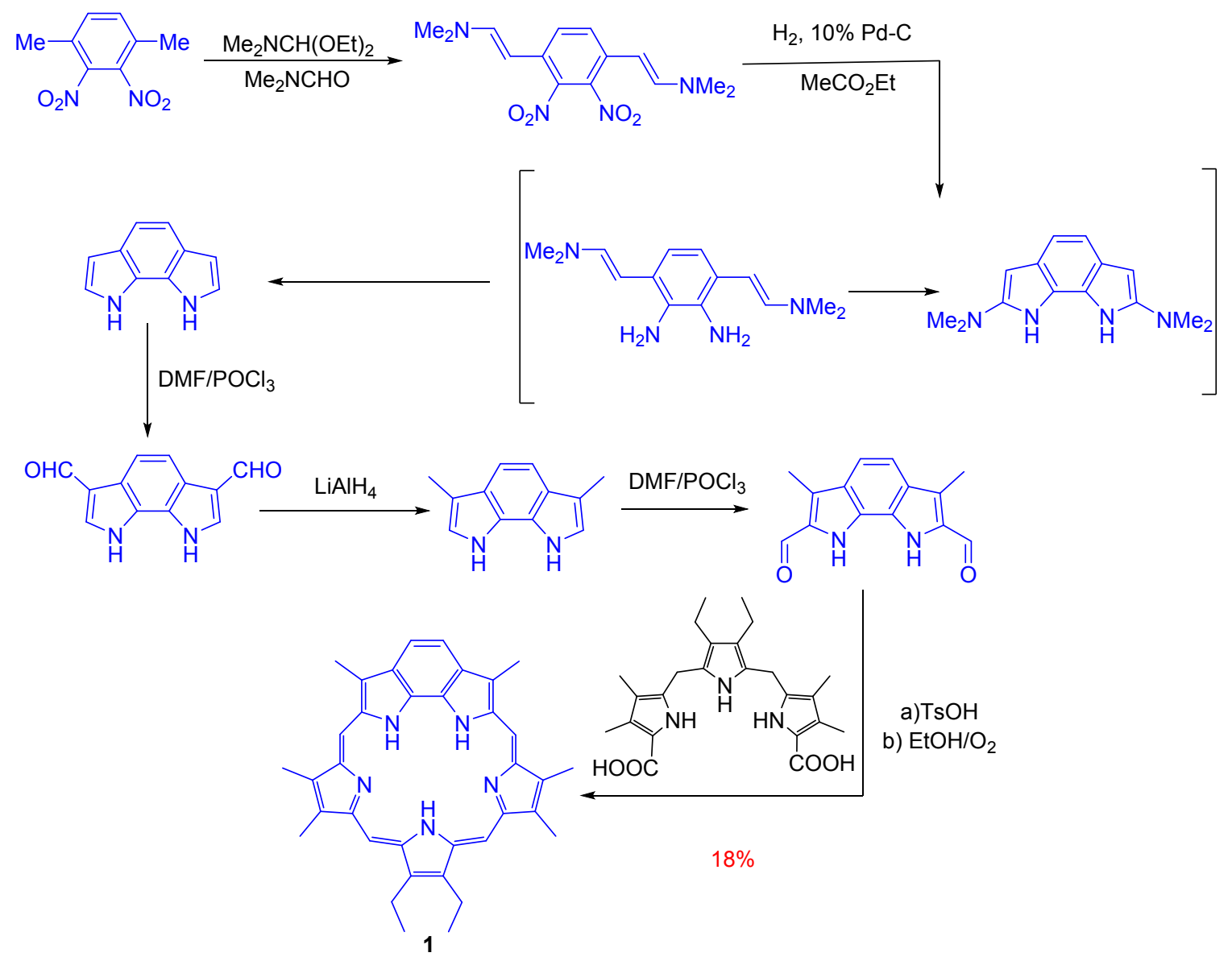

Scheme 1. Synthesis of napthodipyrrole-derived sapphyrin 1. 


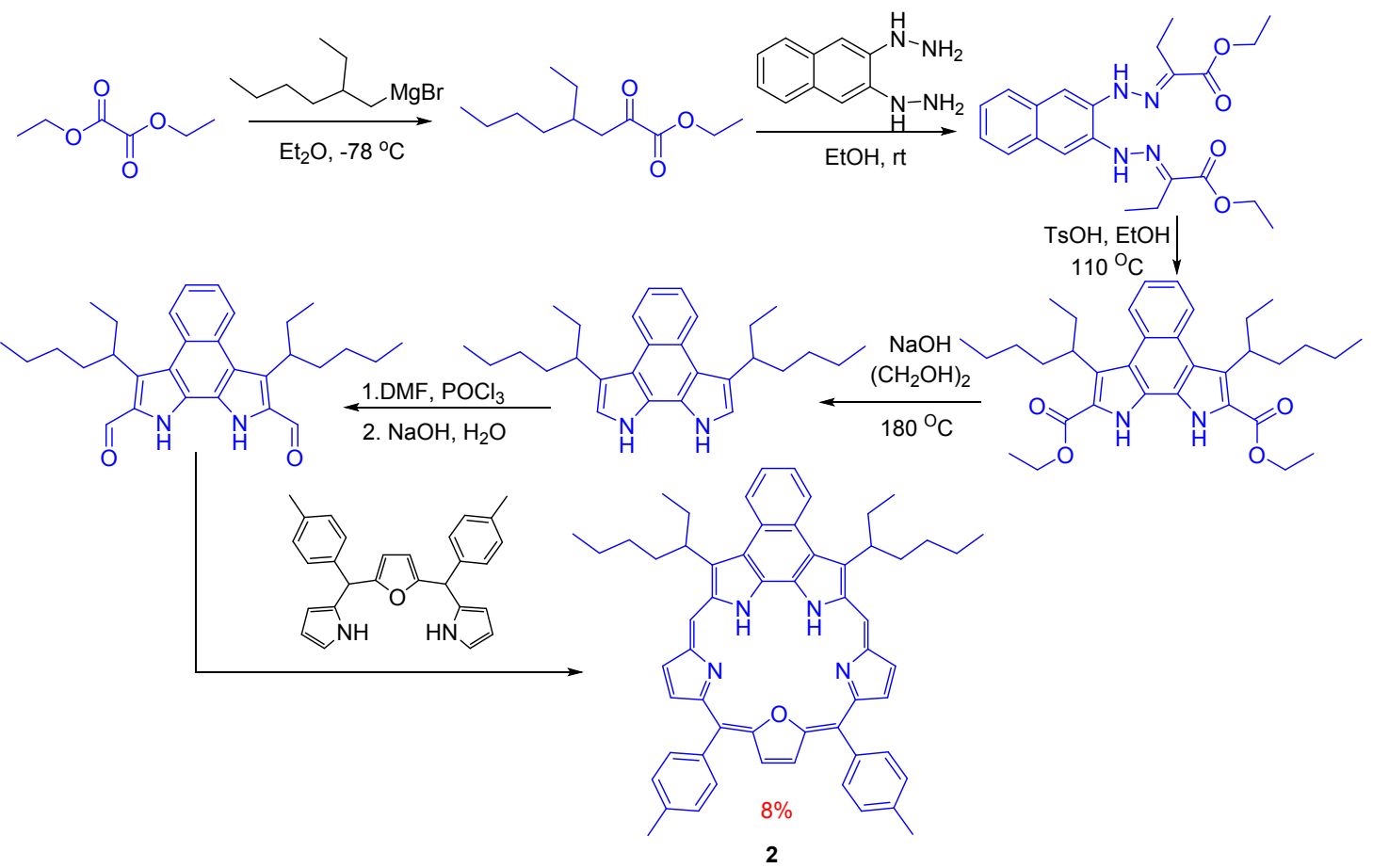

Scheme 2. Synthesis of napthodipyrrole-derived oxasapphyrin 2. 


\section{EXPERIMENTAL SECTION}

Materials and Methods. The chemicals such as $\mathrm{NaBH}_{4}$ and DDQ were used as obtained from Sigma-Aldrich. All other chemicals used for the synthesis were reagent grade unless otherwise specified. The ${ }^{1} \mathrm{H}$ NMR spectra were recorded in deuterated chloroform on 500 and $400 \mathrm{MHz}$ Bruker instruments. The frequencies for the ${ }^{13} \mathrm{C}$ nucleus are 100.06 and $125.77 \mathrm{MHz}$ for 400 and $500 \mathrm{MHz}$ instruments, respectively. Tetramethylsilane was used as an internal standard for ${ }^{1} \mathrm{H}$ and ${ }^{13} \mathrm{C}$ NMR. Absorption and fluorescence spectra were recorded using a SHIMADZU-MPC-3100UV-NIR spectrophotometer and Varian Cary Eclipse spectrofluorometer, respectively. Cyclic voltammetry $(\mathrm{CV})$ studies were performed on the BAS electrochemical system using a threeelectrode configuration consisting of a glassy carbon (working electrode), platinum wire (auxiliary electrode), and saturated calomel (reference electrode). The highresolution mass spectra (HR-MS) were recorded with a Bruker maxis impact 282001.00081 mass spectrometer. Single-crystal X-ray structure analyses were carried out on a Rigaku Saturn724 diffractometer conjugated with a lowtemperature attachment. Data were collected at $100 \mathrm{~K}$ by means of graphitemonochromated Mo K $\alpha$ radiation $(\lambda \alpha=0.71073 \AA)$ by the $\omega$-scan method. The data were reduced by using CrystalClearSM Ex-pert 2.1 b24 software. The structures were solved by direct methods and refined by leastsquares against F2 employing the software packages SHELXL-97, ${ }^{1}$ SIR-92, ${ }^{2}$ and WINGX. ${ }^{3}$ All non-hydrogen atoms were refined anisotropically. CCDC no. 1957306 for macrocycle 3a contain the supplementary crystallographic data of that compound. These data can be obtained free of charge from The Cambridge Crystallographic Data Centre via $\underline{\text { www.ccdc.cam.ac.uk/data_request/cif }}$ 
Computational Details. Full geometry optimization of oxabenzosapphyrin $\mathbf{3 a}$ and its monoprotonated $[\mathbf{3 a . H}]^{+}$and diprotonated $[\mathbf{3 a . 2 H}]^{2+}$ form were carried out by using Gaussian 09 quantum chemical software. ${ }^{4}$ For compound 3a, the density functional theory $(\mathrm{DFT})^{5}$ method with the B3LYP/6-31G(d,p) ${ }^{6}$ basis set was used for all atoms for optimization in $\mathrm{S}_{0}$ state. The frequency calculations were done on $\mathrm{S}_{0}$ optimized geometries to substantiate genuine global minimum energy structures and found that the sum of imaginary frequencies was zero for all the macrocyles. The optimized geometries were used to gain frontier molecular orbitals (FMOs) and were also subjected to single point TD-DFT calculations for the first $50 \mathrm{~S}_{0} \rightarrow \mathrm{S}_{\mathrm{n}}$ transitions to understand absorption properties of macrocycle 3a. ${ }^{7}$ The integral equation formalismpolarizable continuum model (PCM) within the self-consistent reaction field (SCRF) theory was used in the TD-DFT calculations to describe the solvation of $\mathbf{3 a}$ in toluene.

\section{References:}

1. Sheldrick, G. M. Acta Crystallogr. Sect. A Found. Crystallogr. 2008, 64, 112-122.

2. Altomare, A.; Cascarano, G.; Giacovazzo, C.; Guagliardi, A. J. Appl. Crystallogr. 1993, 26, $343-350$.

3. Farrugia, L. J. J. Appl. Crystallogr. 1999, 32, 837-838.

4. Frisch, M. J.; Trucks, G. W.; Schlegel, H. B.; Scuseria, G. E.; Robb, M. A.; Cheeseman, J. R.; Scalmani, G.; Barone, V.; Mennucci, B.; Petersson, G. A.; et al. Gaussian 09 C.01; Gaussian Inc.: Wallingford CT, 2009.

5. Treutler, O.; Ahlrichs, R. J. Chem. Phys. 1995, 102, 346-354.

6. Feller, D. J. Comput. Chem. 1996, 17, 1571-1586.

7. (a) Hehre, W. J. Acc. Chem. Res. 1976, 9, 399-406. (b) Bauernschmitt, R.; Ahlrichs, R. Chem. Phys. Lett. 1996, 256, 454-464. (c) Furche, F.; Rappoport, D. Olivucci, M., Ed.; 
Elsevier: Amsterdam, 2005. (d) Gabe, Y.; Ueno, T.; Urano, Y.; Kojima, H.; Nagano, T. Anal.

Bioanal. Chem. 2006, 386, 621-626. (e) Furche, F.; Ahlrichs, R. J. Chem. Phys. 2002, 117, 7433-7447. (f) Practical Aspects of Computational Chemistry: Methods, Concepts and Applications (Google EBook); Leszczynski, J., Shukla, M., Eds.; Springer, 2009. (g) Scalmani, G.; Frisch, M. J.; Mennucci, B.; Tomasi, J.; Cammi, R.; Barone, V.. J. Chem. Phys. 2006, 124, 094107-094115.

\section{SYNTHESIS}

\section{Method for general synthesis of doubly N-confused dipyrroethene $5 a$ and $5 b$.}

An activated zinc powder (31.2 mmol) and copper (I) chloride (1.04 mmol) in THF (80 $\mathrm{mL}$ ) were taken in a three-necked round bottom flask and purged with nitrogen gas for $10 \mathrm{~min}$. The mixture was cooled to $0{ }^{\circ} \mathrm{C}$, and $\mathrm{TiCl}_{4}(15.6 \mathrm{mmol})$ was added slowly by maintaining the temperature below $0{ }^{\circ} \mathrm{C}$. The suspension was then warmed to room temperature and stirred for 30 min. and refluxed for $3 \mathrm{~h}$. The mixture was again cooled to $0{ }^{\circ} \mathrm{C}$ and the solution of phenyl $(1 \mathrm{H}-$ pyrrol-3-yl)methanone or (1H-pyrrol-3-yl)(p-tolyl)methanone $(5.2 \mathrm{mmol})$ in THF $(25 \mathrm{~mL})$ was added slowly and the reaction mixture was refluxed for $12 \mathrm{~h}$, until the starting material was consumed as judged by TLC analysis. The reaction mixture was quenched with $10 \%$ aqueous $\mathrm{NaHCO}_{3}$ solution and extracted with ether. The organic layers were collected, dried over anhydrous $\mathrm{Na}_{2} \mathrm{SO}_{4}$ and concentrated on rotary evaporator under vacuum. The crude compound was purified by chromatography on silica gel using petroleum ether/ ethyl acetate and afforded desired products $\mathbf{5 a}$ and $\mathbf{5 b}$ in $62-65 \%$ yields as white crystalline solid. 
(E/Z)-1,2-diphenyl-1,2-di(1H-pyrrol-3-yl)ethane 5a: Yield 65\% (1.048 gm), ${ }^{1} \mathrm{H}$ NMR (400 $\mathrm{MHz}, \mathrm{CDCl}_{3}, \delta$ in ppm): $8.40(\mathrm{~s}, 2 \mathrm{H}), 7.83(\mathrm{~s}, 2 \mathrm{H}), 7.41-7.33(\mathrm{~m}, 8 \mathrm{H}), 7.33-7.27(\mathrm{~m}, 4 \mathrm{H}), 7.23$ $(\mathrm{dd}, J=10.0,4.7 \mathrm{~Hz}, 6 \mathrm{H}), 7.19-7.14(\mathrm{~m}, 4 \mathrm{H}), 7.09-7.04(\mathrm{~m}, 2 \mathrm{H}), 6.50(\mathrm{dd}, J=3.0,2.2 \mathrm{~Hz}$, 2H), $6.43(\mathrm{dd}, J=4.9,2.5 \mathrm{~Hz}, 2 \mathrm{H}), 5.91(\mathrm{~d}, J=1.8 \mathrm{~Hz}, 2 \mathrm{H}), 5.32-5.26(\mathrm{~m}, 2 \mathrm{H}) .{ }^{13} \mathrm{C}$ NMR $(100$ $\mathrm{MHz}, \mathrm{CDCl}_{3}, \delta$ in ppm): $145.0,140.5,131.3,130.6,128.3,127.5,126.9,126.3,126.0,125.7$, 124.6, 121.2, 119.3, 116.7, 109.8, 104.7. HRMS (ESI-TOF) m/z: $[\mathrm{M}+\mathrm{H}]^{+}$Calcd. for $\mathrm{C}_{22} \mathrm{H}_{18} \mathrm{~N}_{2}$ 310.1423; Found 311.1542.

(E/Z)-1,2-di(1H-pyrrol-3-yl)-1,2-di-p-tolylethene 5b: Yield 62\% (1.089 gm), ${ }^{1} \mathrm{H}$ NMR (400 $\mathrm{MHz}, \mathrm{CDCl}_{3}, \delta$ in ppm): $8.20(\mathrm{~s}, 2 \mathrm{H}), 7.83(\mathrm{~s}, 1 \mathrm{H}), 7.27(\mathrm{~s}, 1 \mathrm{H}), 7.16(\mathrm{~d}, J=7.9 \mathrm{~Hz}, 4 \mathrm{H}), 7.04(\mathrm{~d}$, $J=7.8 \mathrm{~Hz}, 4 \mathrm{H}), 7.02-6.92(\mathrm{~m}, 2 \mathrm{H}), 6.53-6.46(\mathrm{~m}, 2 \mathrm{H}), 6.43(\mathrm{~d}, J=2.4 \mathrm{~Hz}, 1 \mathrm{H}), 5.94(\mathrm{~d}, J=$ $1.6 \mathrm{~Hz}, 1 \mathrm{H}), 5.34(\mathrm{~d}, J=1.4 \mathrm{~Hz}, 1 \mathrm{H}), 2.40(\mathrm{~s}, 1 \mathrm{H}), 2.33(\mathrm{~s}, 6 \mathrm{H}) .{ }^{13} \mathrm{C} \mathrm{NMR}\left(100 \mathrm{MHz}, \mathrm{CDCl}_{3}, \delta\right.$ in ppm): $137.5,135.0,131.1,130.4,129.0,128.3,127.6,125.7,124.8,121.3,104.5,21.2 . \mathrm{HRMS}$ (ESI-TOF) m/z: $[\mathrm{M}+\mathrm{H}]^{+}$Calcd. for $\mathrm{C}_{24} \mathrm{H}_{22} \mathrm{~N}_{2}$ 338.1783; Found 339.1851.

\section{General Procedure for the Preparation of Compounds 6a-6c.}

Freshly prepared ethyl magnesium bromide in THF (20 mL, $10.64 \mathrm{mmol}, 0.53 \mathrm{M})$ was added dropwise to the solution of the desired 5,6-diaryl dipyrroethene (E and $\mathrm{Z}$ mixture) (1.77 $\mathrm{mmol})$ in toluene $(20 \mathrm{~mL})$ under nitrogen. The resulting solution was stirred for $15-20 \mathrm{~min}$ at room temperature, and then the corresponding aryl acid chloride $(4.3 \mathrm{mmol})$ was added to this at $0{ }^{\circ} \mathrm{C}$. The resulting mixture was stirred for 20-30 min at room temperature. The reaction mixture was quenched with an ice-cold solution of $\mathrm{NH}_{4} \mathrm{Cl}(100 \mathrm{~mL})$ and extracted with ethyl acetate $(3 \times 50$ $\mathrm{mL}$ ). The combined organic layers were dried over anhydrous $\mathrm{Na}_{2} \mathrm{SO}_{4}$. The yellowish brown semisolid obtained after removal of solvent under vacuo was subjected to silica gel (60-120) 
column chromatography using petroleum ether/ethyl acetate (80:20) and afforded the compounds 6a-6c as $\mathrm{E}$ and $\mathrm{Z}$ mixtures in $67-78 \%$ yields as light yellow amorphous solid.

(E/Z)-((1,2-diphenylethene-1,2-diyl)bis(1H-pyrrole-4,2-diyl))bis(p-tolylmethanone) 6a: 78\% yield (753 mg), ${ }^{1} \mathrm{H}$ NMR (500 MHz, $\mathrm{CDCl}_{3}$ ): 9.46 (s, 2H), $9.12(\mathrm{~s}, 2 \mathrm{H}), 7.54(\mathrm{~d}, J=8.1 \mathrm{~Hz}, 4 \mathrm{H})$, $7.42(\mathrm{~d}, J=8.1 \mathrm{~Hz}, 4 \mathrm{H}), 7.18-7.14(\mathrm{~m}, 10 \mathrm{H}), 7.14-7.07(\mathrm{~m}, 10 \mathrm{H}), 7.05(\mathrm{~d}, J=8.0 \mathrm{~Hz}, 4 \mathrm{H})$, $6.94(\mathrm{dd}, J=6.5,3.1 \mathrm{~Hz}, 4 \mathrm{H}), 6.84(\mathrm{t}, J=2.8 \mathrm{~Hz}, 2 \mathrm{H}), 6.44(\mathrm{dd}, J=2.9,1.3 \mathrm{~Hz}, 2 \mathrm{H}), 6.16-6.11$ (m, 2H), $5.96(\mathrm{t}, J=2.7 \mathrm{~Hz}, 3 \mathrm{H}), 2.39(\mathrm{~s}, 6 \mathrm{H}), 2.19(\mathrm{~s}, 6 \mathrm{H}) .{ }^{13} \mathrm{C} \mathrm{NMR}\left(100 \mathrm{MHz}, \mathrm{CDCl}_{3}, \delta\right.$ in ppm): 185.9, 184.4, 143.9, 143.3, 142.3, 142.2, 135.9, 135.2, 133.3, 132.0, 130.9, 130.7, 129.5, 129.1, 128.8, 128.4, 127.7, 127.3, 126.7, 126.2, 122.5, 121.7, 114.7, 21.6, 21.4. HRMS (ESI-TOF) m/z: $[\mathrm{M}+\mathrm{H}]^{+}$Calcd. for $\mathrm{C}_{38} \mathrm{H}_{30} \mathrm{~N}_{2} \mathrm{O}_{2}$ 546.2370; Found 547.2348.

(E/Z)-((1,2-di-p-tolylethene-1,2-diyl)bis(1H-pyrrole-4,2-diyl))bis(p-tolylmethanone) 6b: 67\% yield (680 mg), mp 100-102 ${ }^{\circ} \mathrm{C} ;{ }^{1} \mathrm{H}$ NMR $\left(500 \mathrm{MHz}, \mathrm{CDCl}_{3}\right): 9.82$ (s, 2H), 9.41 (s, 2H), 7.54 (d, $J=8.1 \mathrm{~Hz}, 4 \mathrm{H}), 7.43(\mathrm{~d}, J=8.1 \mathrm{~Hz}, 4 \mathrm{H}), 7.13(\mathrm{~d}, J=7.9 \mathrm{~Hz}, 4 \mathrm{H}), 7.08-6.99(\mathrm{~m}, 8 \mathrm{H}), 6.96(\mathrm{~d}, J$ $=7.9 \mathrm{~Hz}, 4 \mathrm{H}), 6.88(\mathrm{~d}, J=7.9 \mathrm{~Hz}, 4 \mathrm{H}), 6.85(\mathrm{t}, J=2.8 \mathrm{~Hz}, 2 \mathrm{H}), 6.81-6.75(\mathrm{~m}, 4 \mathrm{H}), 6.45(\mathrm{dd}, J$ $=3.0,1.4 \mathrm{~Hz}, 2 \mathrm{H}), 6.14(\mathrm{dd}, J=2.5,1.5 \mathrm{~Hz}, 2 \mathrm{H}), 5.97(\mathrm{t}, J=2.7 \mathrm{~Hz}, 2 \mathrm{H}), 2.40(\mathrm{~s}, 6 \mathrm{H}), 2.35(\mathrm{~s}$, 6H), 2.25 (s, 6H), 2.15 (s, 6H). ${ }^{13} \mathrm{C} \mathrm{NMR} \mathrm{(100} \mathrm{MHz,} \mathrm{CDCl}_{3}, \delta$ in ppm): 186.1, 184.4, 142.2, 142.0, $141.2,140.3,136.09,135.7,135.4,134.6,133.7,131.5,130.7,130.6,130.4,129.7,129.49,129.1$, 129.0, 128.9, 128.6, 128.2, 128.1, 127.9, 127.1, 122.6, 122.1, 114.5, 21.5, 21.3, 21.2, 21.1. HRMS (ESI-TOF) m/z: [M+H] $]^{+}$Calcd. for $\mathrm{C}_{24} \mathrm{H}_{19} \mathrm{~N}_{2}$ 574.2620; Found 573.2548.

(E/Z)-((1,2-diphenylethene-1,2-diyl)bis(1H-pyrrole-4,2-diyl))bis(phenylmethanone) 6c: 71\% yield $(679.8 \mathrm{mg}), \mathrm{mp} 100-102{ }^{\circ} \mathrm{C} ;{ }^{1} \mathrm{H} \mathrm{NMR}\left(500 \mathrm{MHz}, \mathrm{CDCl}_{3}\right): 9.50(\mathrm{~s}, 2 \mathrm{H}), 9.18(\mathrm{~s}, 1 \mathrm{H}), 7.64-$ $7.60(\mathrm{~m}, 2 \mathrm{H}), 7.49$ (ddt, $J=8.6,7.0,2.8 \mathrm{~Hz}, 10 \mathrm{H}), 7.41-7.37(\mathrm{~m}, 8 \mathrm{H}), 7.36-7.30(\mathrm{~m}, 8 \mathrm{H}), 7.27$ (d, $J=7.7 \mathrm{~Hz}, 2 \mathrm{H}), 7.18-7.04(\mathrm{~m}, 10 \mathrm{H}), 6.98-6.92(\mathrm{~m}, 2 \mathrm{H}), 6.90-6.78(\mathrm{~m}, 1 \mathrm{H}), 6.48-6.37$ 
$(\mathrm{m}, 2 \mathrm{H}), 6.12(\mathrm{dt}, J=19.1,9.5 \mathrm{~Hz}, 1 \mathrm{H}), 6.05-5.99(\mathrm{~m}, 2 \mathrm{H}), 5.96(\mathrm{t}, J=2.7 \mathrm{~Hz}, 1 \mathrm{H}) .{ }^{13} \mathrm{C} \mathrm{NMR}$

(100 MHz, $\mathrm{CDCl}_{3}, \delta$ in ppm): 186.0, 184.6, 184.6, 143.9, 143.7, 143.2, 138.6, 137.9, 137.8, 135.0, $133.6,132.2,131.7,131.6,131.3,130.8,130.6,130.6,130.2,130.0,129.8,129.3,129.0,129.0$, $128.8,128.8,128.7,128.1,127.7,127.4,127.1,127.0,126.9,126.8,126.8,126.2,122.8,121.8$, 120.5, 114.9. HRMS (ESI-TOF) $\mathrm{m} / \mathrm{z}$ : $[\mathrm{M}+\mathrm{Na}]^{+}$Calcd. for $\mathrm{C}_{36} \mathrm{H}_{26} \mathrm{~N}_{2} \mathrm{O}_{2} \mathrm{Na}$ 541.1892; Found 541.1887.

\section{General Procedure for Synthesis of Benzooxasapphyrin 3a-3c.}

To the solution of the appropriate diacylated dipyrroethene $\mathbf{6 a - 6 c}(0.18 \mathrm{mmol})$ in $10 \mathrm{~mL}$ of THF/MeOH (9:1 v/v) was added $\mathrm{NaBH}_{4}(3.66 \mathrm{mmol})$ under a $\mathrm{N}_{2}$ atmosphere. The reaction was stirred at room temperature for 15-20 min under a $\mathrm{N}_{2}$ atmosphere. After completion of the reaction, as indicated by TLC analysis, the reaction was quenched with a saturated aqueous solution of $\mathrm{NH}_{4} \mathrm{Cl}(50 \mathrm{~mL})$ and extracted with diethyl ether $(2 \times 50 \mathrm{~mL})$. The combined organic layers were dried over anhydrous sodium sulfate, and the solvent was removed on a rotary evaporator under a high vacuum to afford dicarbinols as yellow sticky solids. The appropriate crude dicarbinol without further purification was treated with 1 equiv of p-tolyloxatripyrrane $(0.18$ $\mathrm{mmol})$ in the presence of a catalytic amount of TFA $(0.5$ equiv, $0.018 \mathrm{mmol})$ in $100 \mathrm{~mL}$ of $\mathrm{CH}_{2} \mathrm{Cl}_{2}$ under a $\mathrm{N}_{2}$ atmosphere for $30 \mathrm{~min}$. DDQ $(0.457 \mathrm{mmol})$ was added to the reaction mixture, and stirring was continued for an additional $30 \mathrm{~min}$ in open air. TLC analysis showed the formation of one major orange fluorescent spot along with 2-3 more polar minor spots. The reaction mixture was subjected to basic alumina column chromatography using petroleum ether/ $\mathrm{CH}_{2} \mathrm{Cl}_{2}(85: 15)$ and afforded the corresponding oxabenzosapphyrin 3a-3c as brown red crystalline solids in $8-10 \%$ yields. 
Compound 3a: 10\% yield (16.4 mg), ${ }^{1} \mathrm{H}$ NMR (500 MHz, $\left.\mathrm{CDCl}_{3}\right): 8.60(\mathrm{~s}, 2 \mathrm{H}), 8.42-8.20$ (m, 4H), $8.08(\mathrm{~d}, J=8.0 \mathrm{~Hz}, 4 \mathrm{H}), 8.04(\mathrm{~d}, J=7.5 \mathrm{~Hz}, 2 \mathrm{H}), 7.99(\mathrm{~d}, J=7.6 \mathrm{~Hz}, 2 \mathrm{H}), 7.63(\mathrm{~s}, 4 \mathrm{H}), 7.51$ (t, $J=8.7 \mathrm{~Hz}, 8 \mathrm{H}), 7.40(\mathrm{t}, J=7.4 \mathrm{~Hz}, 4 \mathrm{H}), 7.35(\mathrm{~d}, J=7.2 \mathrm{~Hz}, 2 \mathrm{H}), 3.65(\mathrm{~s}, 2 \mathrm{H}), 2.62(\mathrm{~s}, 6 \mathrm{H})$, $2.55(\mathrm{~s}, 6 \mathrm{H}), 1.40(\mathrm{~s}, 2 \mathrm{H}) .{ }^{13} \mathrm{C} \mathrm{NMR}\left(100 \mathrm{MHz}, \mathrm{CDCl}_{3}, \delta\right.$ in ppm): 160.4, 150.7, 139.6, 139.8, $137.9,136.9,135.3,134.7,134.5,134.4,134.3,133.7,131.7,131.4,130.8,130.2,129.8,129.6$, 129.3, 128.1, 127.8, 127.4, 126.6, 122.1, 120.3, 117.9, 21.4, 21.4. HRMS (ESI-TOF) m/z: [M+H] ${ }^{+}$ Calcd. for $\mathrm{C}_{66} \mathrm{H}_{48} \mathrm{~N}_{4} \mathrm{O}$ 912.3828; Found 913.3900.

Compound 3b: 8\% yield (13.5 mg), ${ }^{1} \mathrm{H}$ NMR (500 MHz, $\left.\mathrm{CDCl}_{3}\right): 8.64(\mathrm{~s}, 2 \mathrm{H}), 8.38-8.26(\mathrm{~m}$, 4H), $8.09(\mathrm{~d}, J=8.0 \mathrm{~Hz}, 4 \mathrm{H}), 8.05(\mathrm{~d}, J=7.5 \mathrm{~Hz}, 2 \mathrm{H}), 8.00(\mathrm{~d}, J=7.5 \mathrm{~Hz}, 2 \mathrm{H}), 7.57-7.42(\mathrm{~m}$, 12H), $7.21(\mathrm{~d}, J=7.8 \mathrm{~Hz}, 4 \mathrm{H}), 3.65(\mathrm{~s}, 2 \mathrm{H}), 2.63(\mathrm{~s}, 6 \mathrm{H}), 2.55(\mathrm{~s}, 6 \mathrm{H}), 2.45(\mathrm{~s}, 6 \mathrm{H}), 1.32(\mathrm{~s}, 2 \mathrm{H})$. ${ }^{13} \mathrm{C} \mathrm{NMR}\left(100 \mathrm{MHz}, \mathrm{CDCl}_{3}, \delta\right.$ in ppm): 160.4, 160.3, 150.7, 137.8, 136.8, 136.7, 135.8, 135.3, $134.8,134.4,134.3,133.7,131.6,131.5,130.8,130.6,130.6,129.7,129.3,129.2,128.8,128.6$, 128.2, 122.2, 120.5, 117.7, 21.4, 21.4, 21.3. HRMS (ESI-TOF) m/z: $[\mathrm{M}+\mathrm{H}]^{+}$Calcd. for $\mathrm{C}_{68} \mathrm{H}_{52} \mathrm{~N}_{4} \mathrm{O}$ 940.4141; Found 941.4219.

Compound 3c: $8 \%$ yield (12.7 mg), ${ }^{1} \mathrm{H}$ NMR (500 MHz, $\left.\mathrm{CDCl}_{3}\right): 8.60(\mathrm{~s}, 2 \mathrm{H}), 8.38(\mathrm{~d}, J=4.4$ $\mathrm{Hz}, 2 \mathrm{H}), 8.35(\mathrm{~d}, J=4.4 \mathrm{~Hz}, 2 \mathrm{H}), 8.16(\mathrm{~d}, J=6.9 \mathrm{~Hz}, 2 \mathrm{H}), 8.09(\mathrm{~d}, J=8.0 \mathrm{~Hz}, 6 \mathrm{H}), 7.68(\mathrm{dd}, J=$ 17.9, 13.4 Hz, 10H), $7.52(\mathrm{~d}, J=7.9 \mathrm{~Hz}, 4 \mathrm{H}), 7.39$ (t, $J=7.4 \mathrm{~Hz}, 4 \mathrm{H}), 7.33$ (t, $J=7.3 \mathrm{~Hz}, 2 \mathrm{H})$, $3.48(\mathrm{~s}, 2 \mathrm{H}), 2.55(\mathrm{~s}, 6 \mathrm{H}), 1.39(\mathrm{~s}, 2 \mathrm{H}) .{ }^{13} \mathrm{C} \mathrm{NMR}\left(100 \mathrm{MHz}, \mathrm{CDCl}_{3}, \delta\right.$ in ppm): 160.6, 160.4, $150.7,139.6,139.5,136.9,135.3,134.7,134.5,134.4,134.3,133.7,131.7,131.4,130.8,130.2$, 129.8, 129.6, 129.3, 128.1, 127.8, 127.4, 126.6, 122.1, 120.3, 117.9, 21.4. HRMS (ESI-TOF) m/z: $[\mathrm{M}+\mathrm{H}]^{+}$Calcd. for $\mathrm{C}_{64} \mathrm{H}_{44} \mathrm{~N}_{4} \mathrm{O}$ 884.3515; Found 885.3655. 

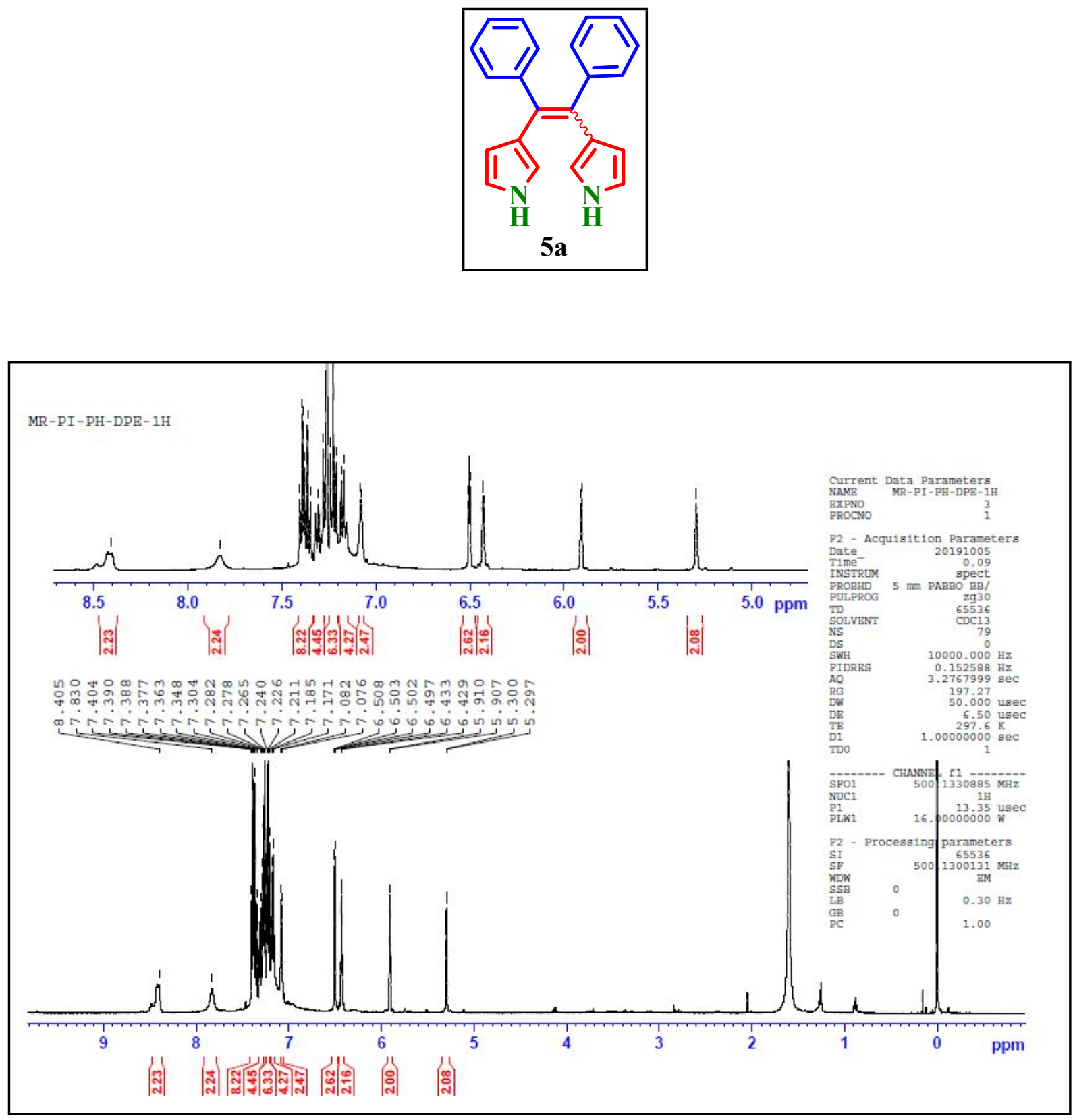

Figure S1. ${ }^{1} \mathrm{H}$ NMR of compound 5 a recorded in $\mathrm{CDCl}_{3}$. 


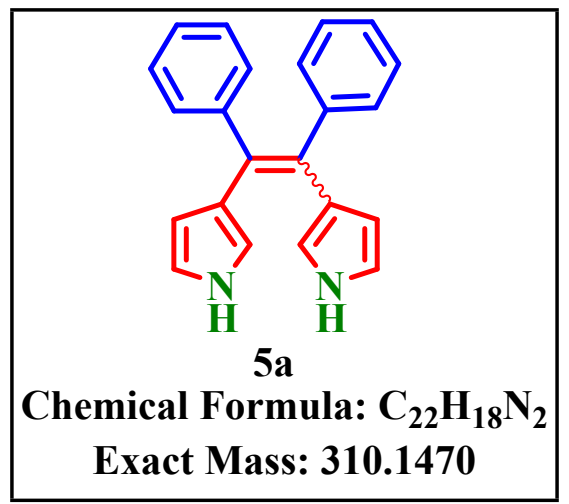

Observed Mass $=311.1542[\mathrm{M}+\mathrm{H}]^{+}$

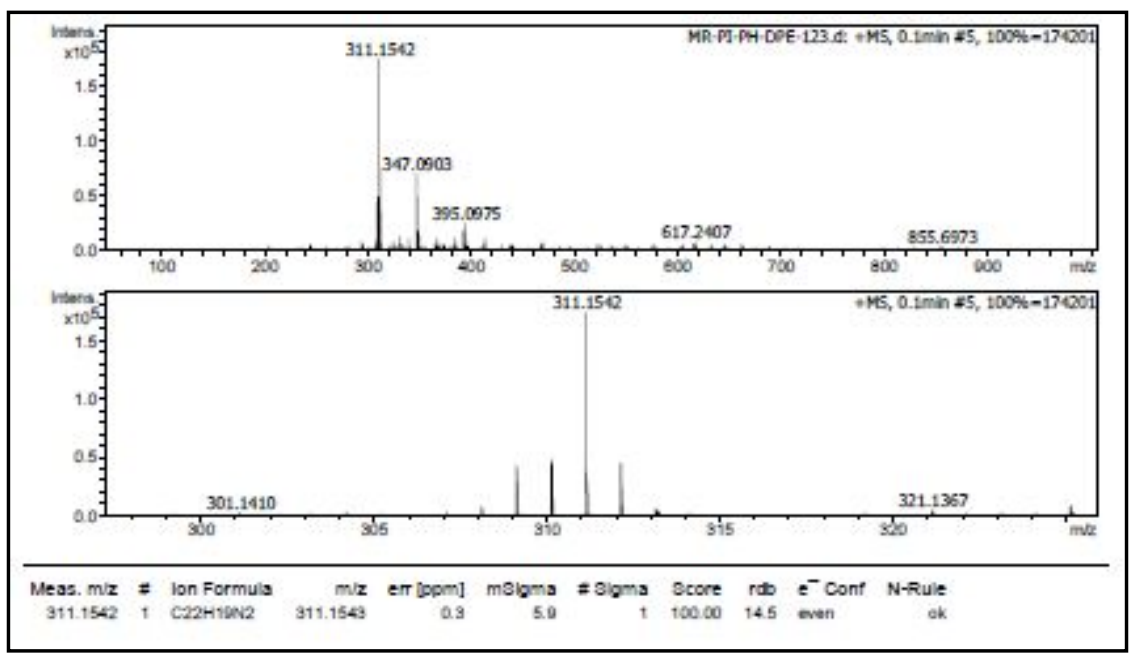

Figure S2. HRMS spectrum of compound 5a. 

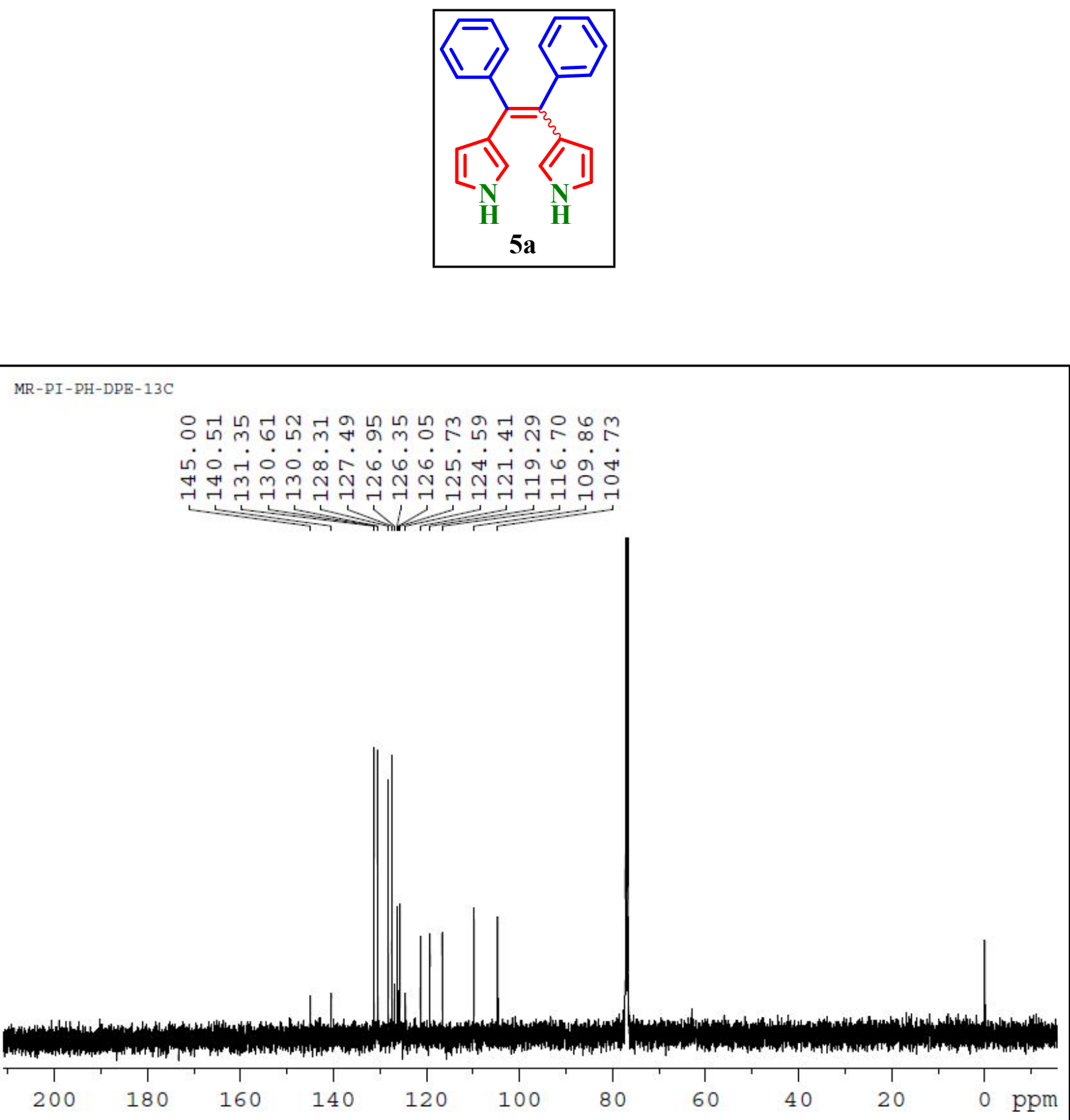

Figure S3. ${ }^{13} \mathrm{C}$ NMR of compound 5a recorded in $\mathrm{CDCl}_{3}$. 

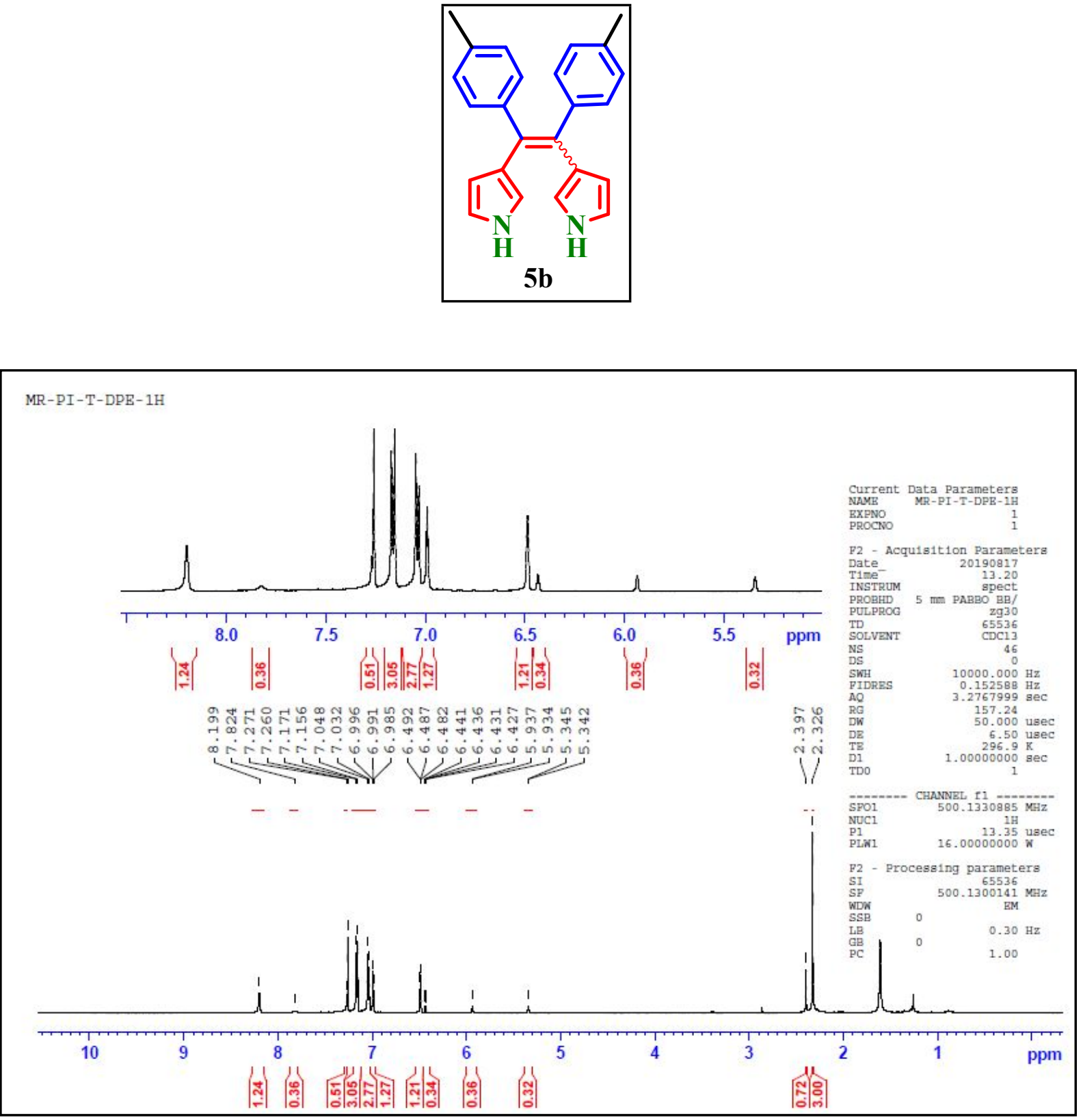

Figure S4. ${ }^{1} \mathrm{H}$ NMR of compound $\mathbf{5 b}$ recorded in $\mathrm{CDCl}_{3}$. 

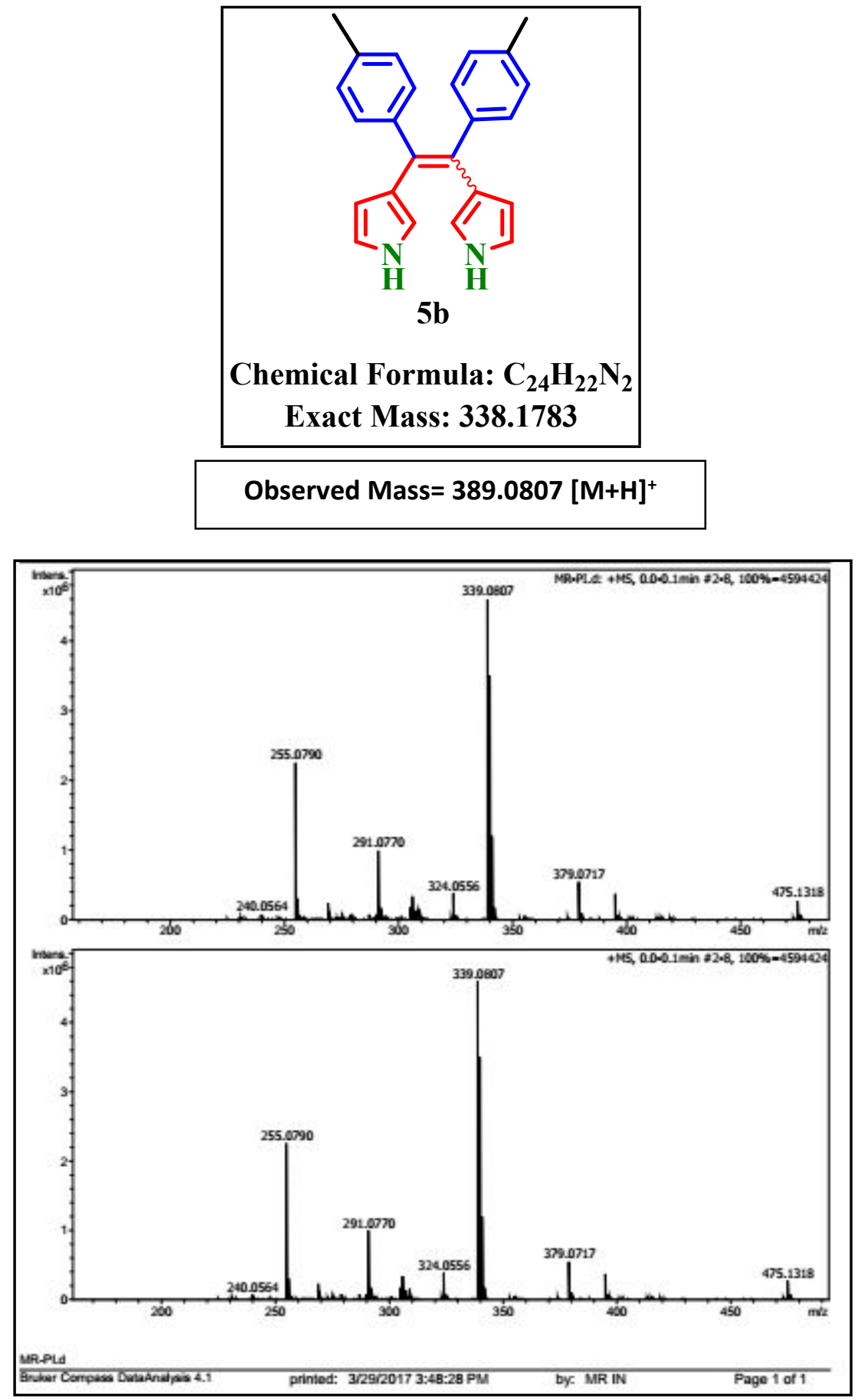

Figure S5. HRMS spectrum of compound $\mathbf{5 b}$. 

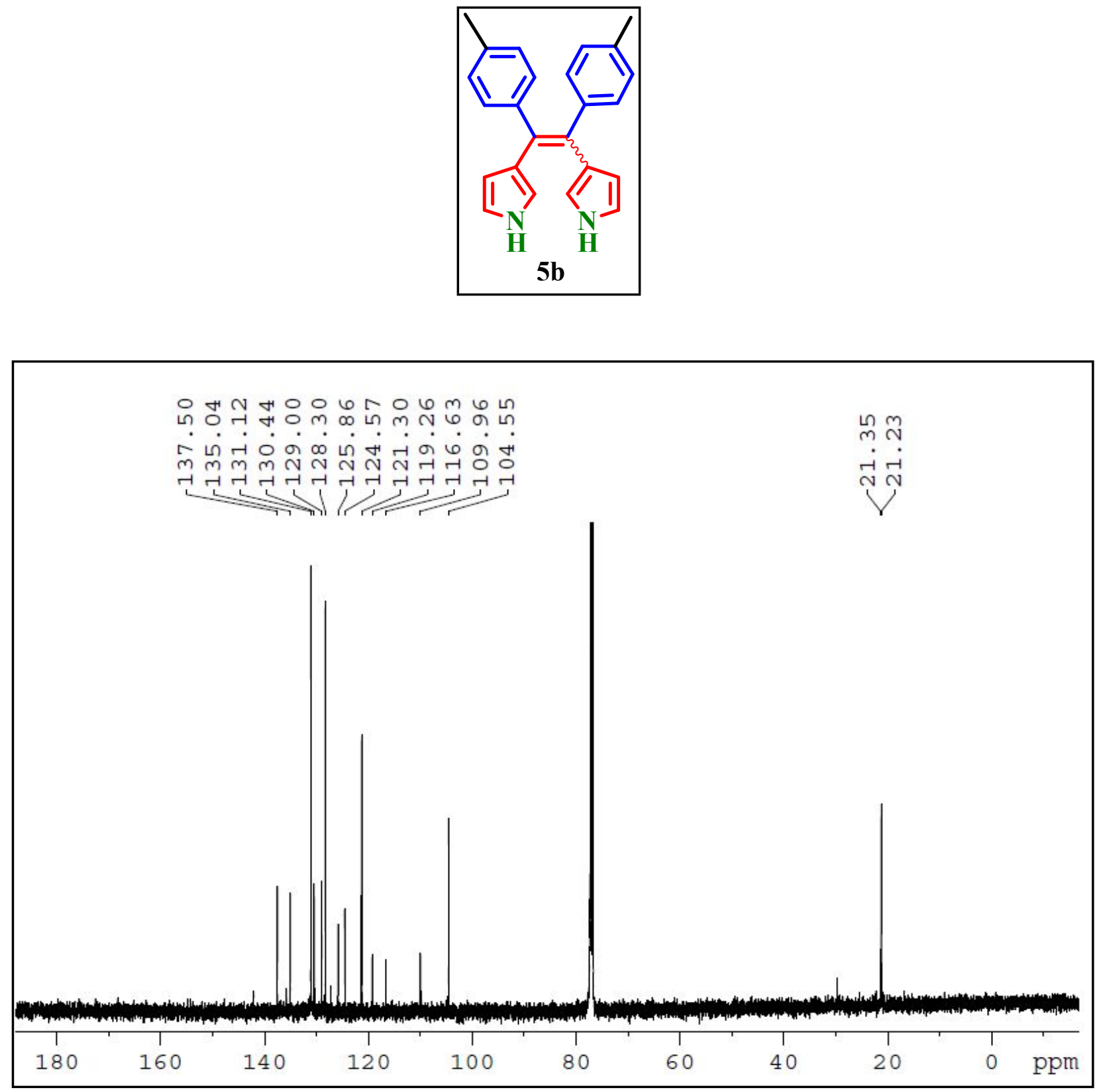

Figure S6. ${ }^{13} \mathrm{C}$ NMR of compound $\mathbf{5 b}$ recorded in $\mathrm{CDCl}_{3}$. 

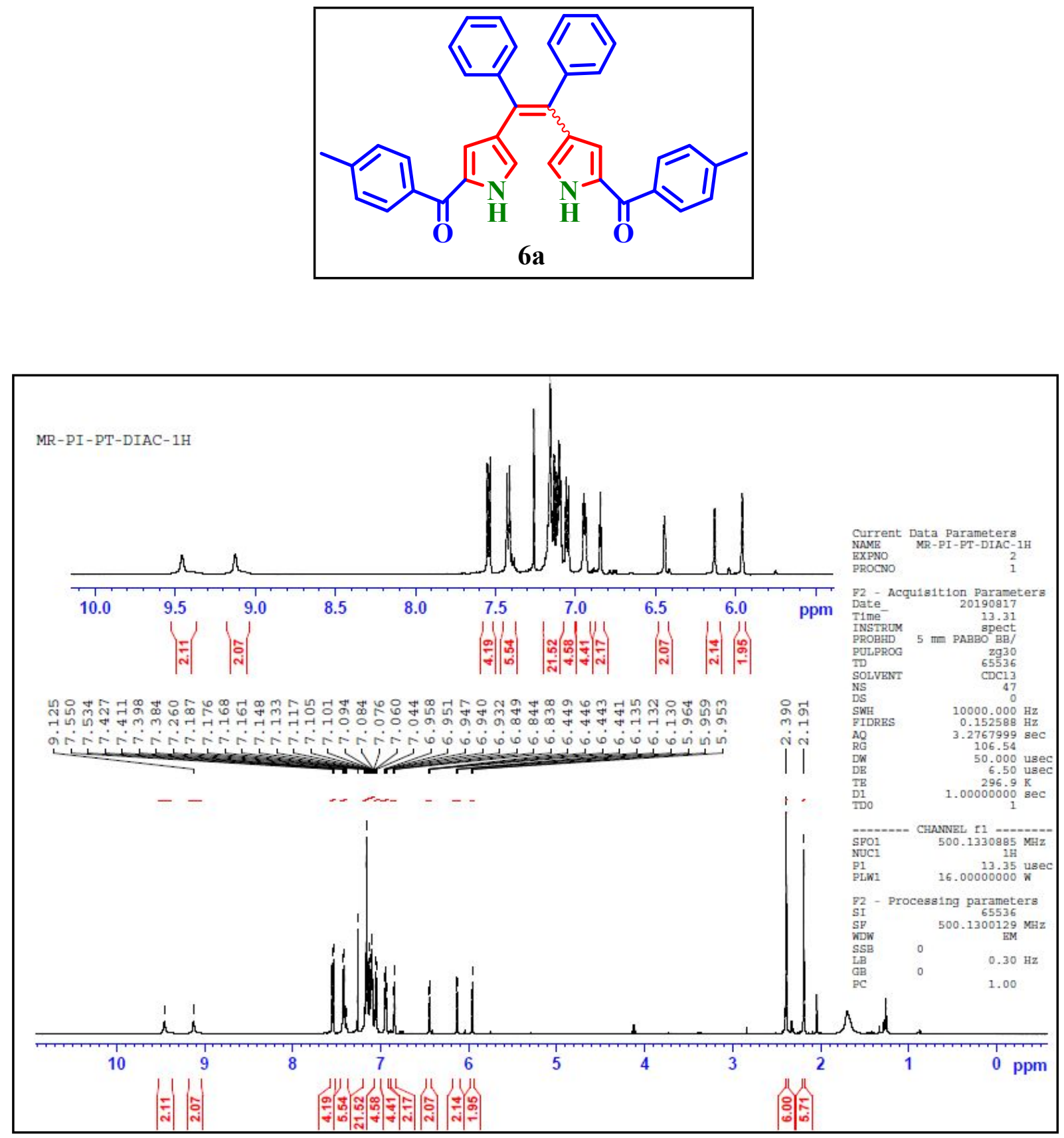

Figure S7. ${ }^{1} \mathrm{H}$ NMR of compound $\mathbf{6 a}$ recorded in $\mathrm{CDCl}_{3}$. 


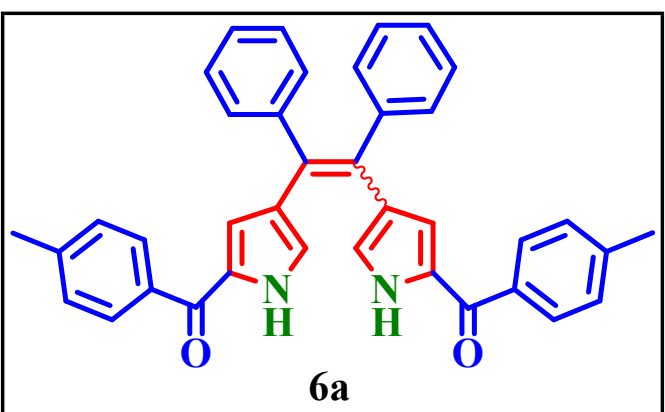

Chemical Formula: $\mathrm{C}_{38} \mathrm{H}_{30} \mathrm{~N}_{2} \mathrm{O}_{2}$ Exact Mass: 546.2307

\section{Observed Mass $=547.2384[\mathrm{M}+\mathrm{H}]^{+}$}

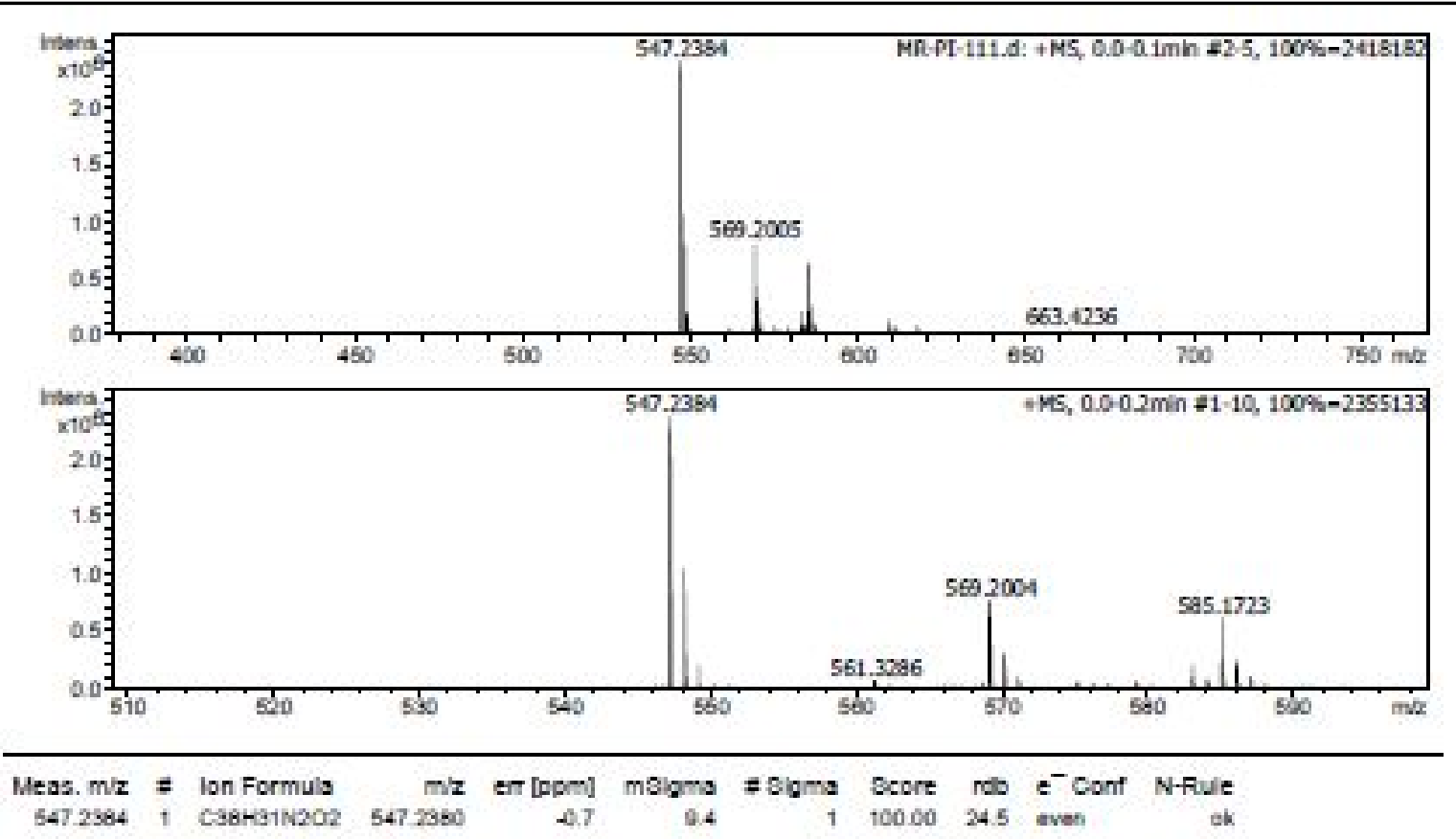

Figure S8. HRMS spectrum of compound $6 \mathbf{a}$. 

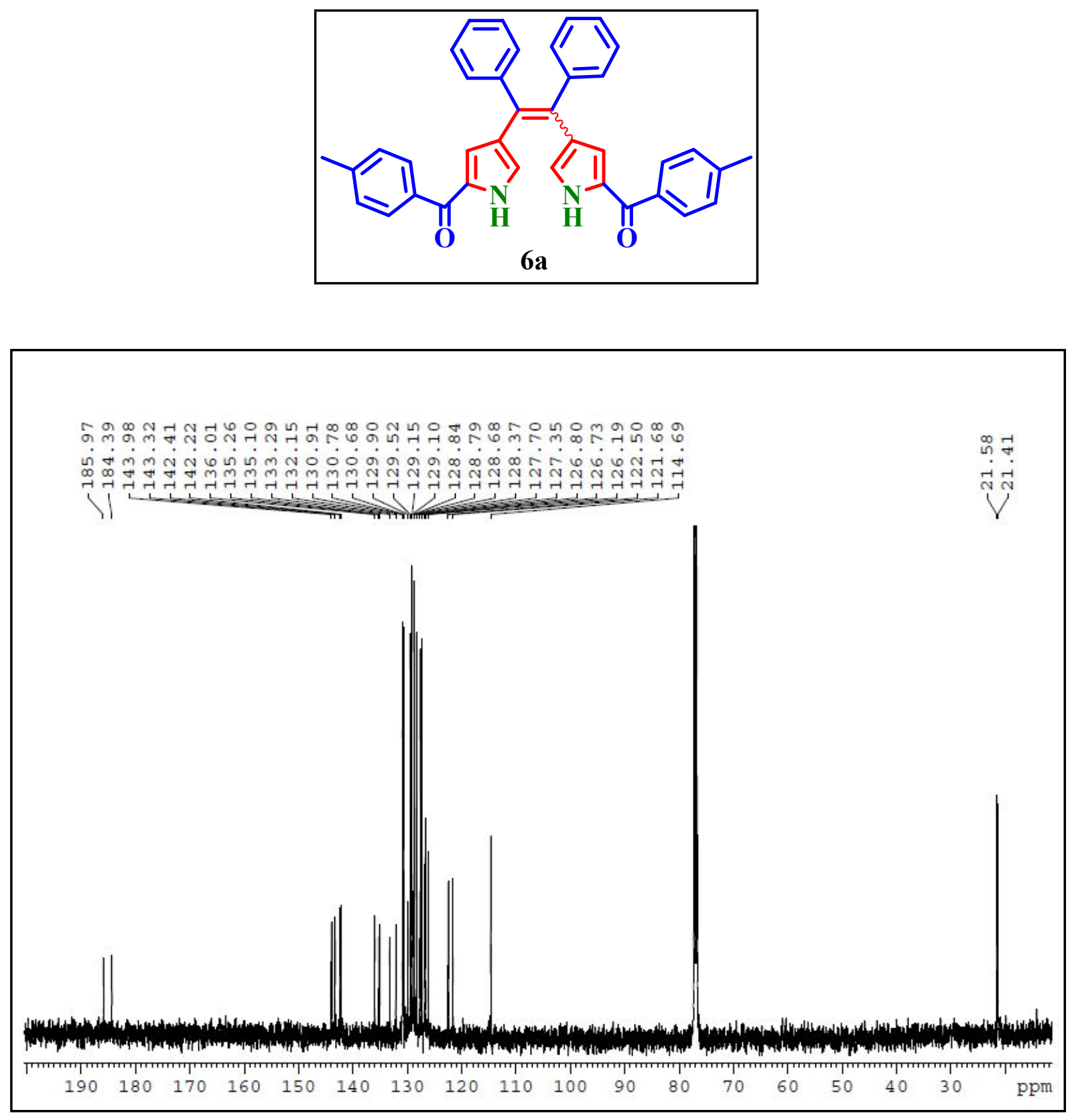

Figure S9. ${ }^{13} \mathrm{C} \mathrm{NMR}$ of compound 6a recorded in $\mathrm{CDCl}_{3}$. 

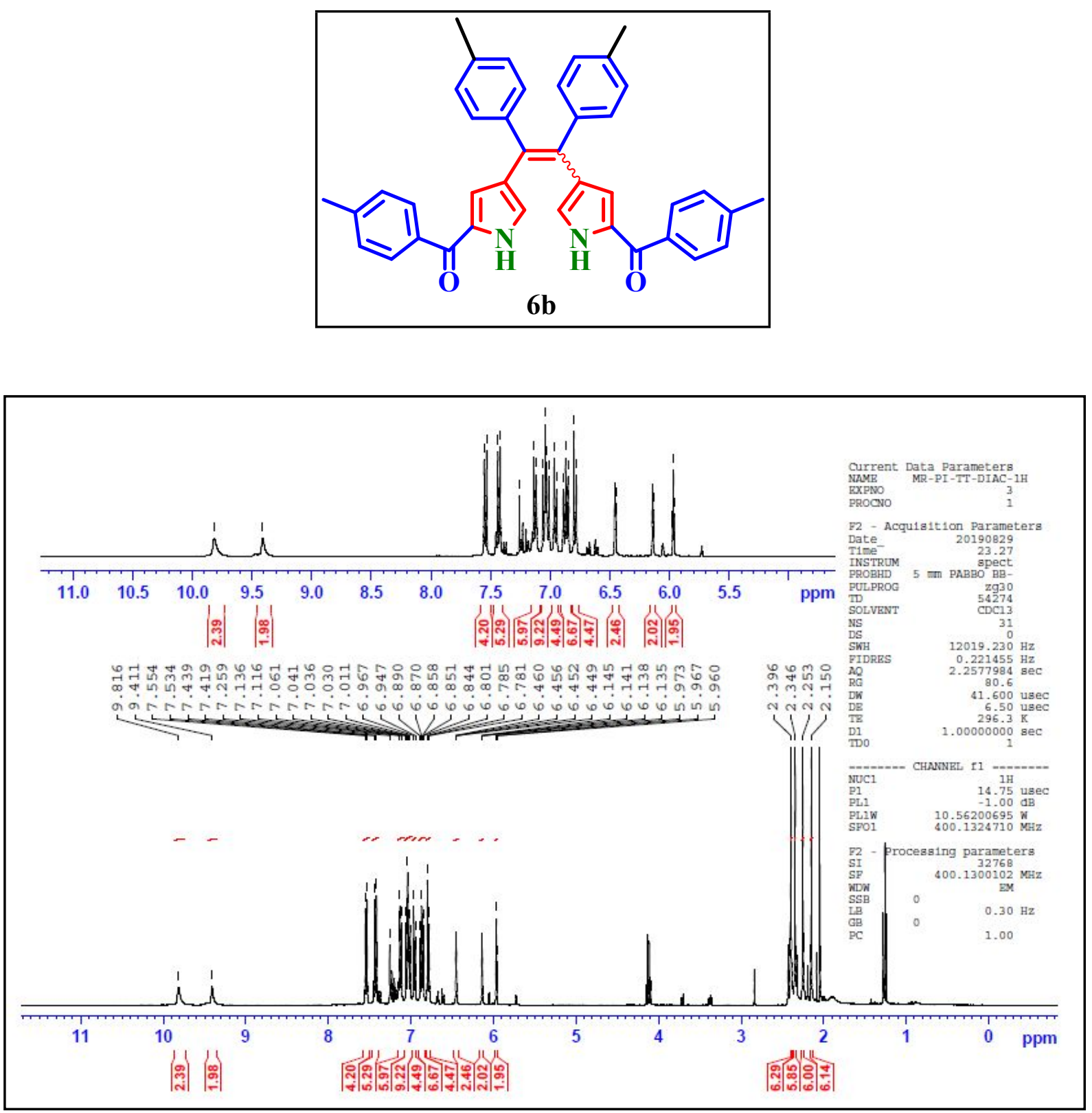

Figure S10. ${ }^{1} \mathrm{H}$ NMR of compound $\mathbf{6 b}$ recorded in $\mathrm{CDCl}_{3}$. 


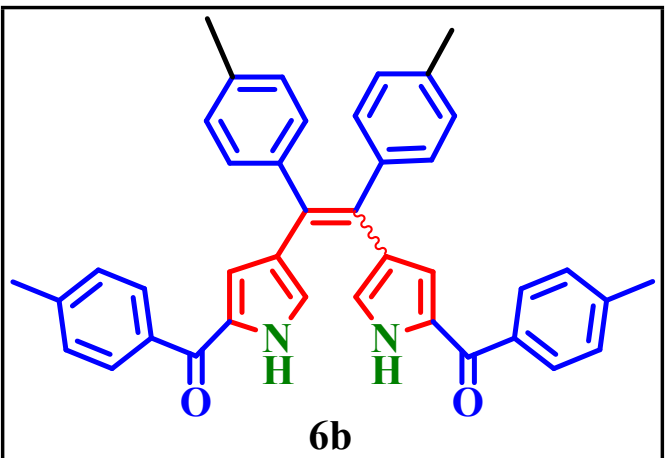

Chemical Formula: $\mathrm{C}_{40} \mathrm{H}_{34} \mathrm{~N}_{2} \mathrm{O}_{2}$

Exact Mass: 574.2620

Observed Mass= $573.2548[\mathrm{M}-\mathrm{H}]^{+}$

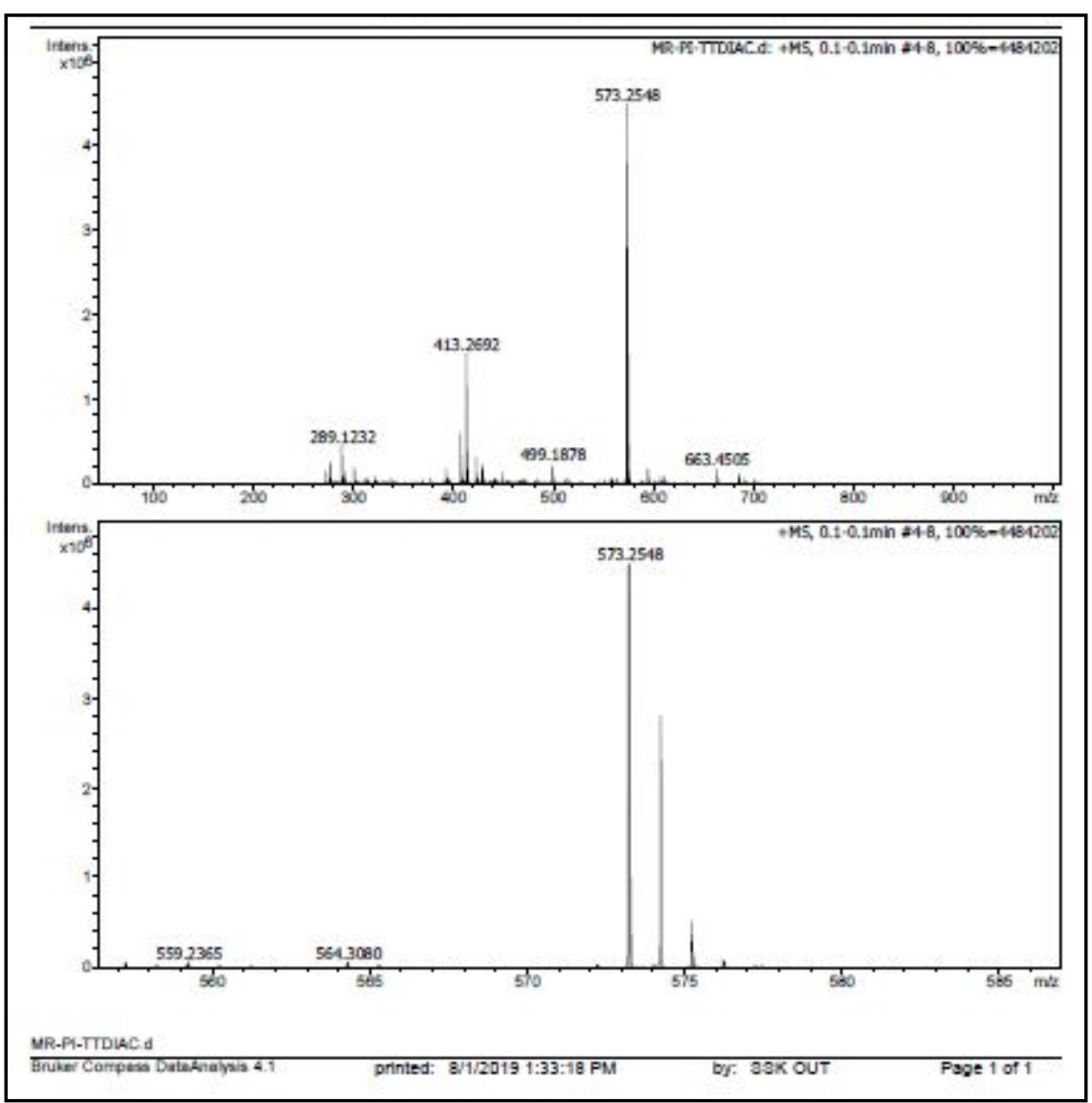

Figure S11. HRMS spectrum of compound $\mathbf{6 b}$. 

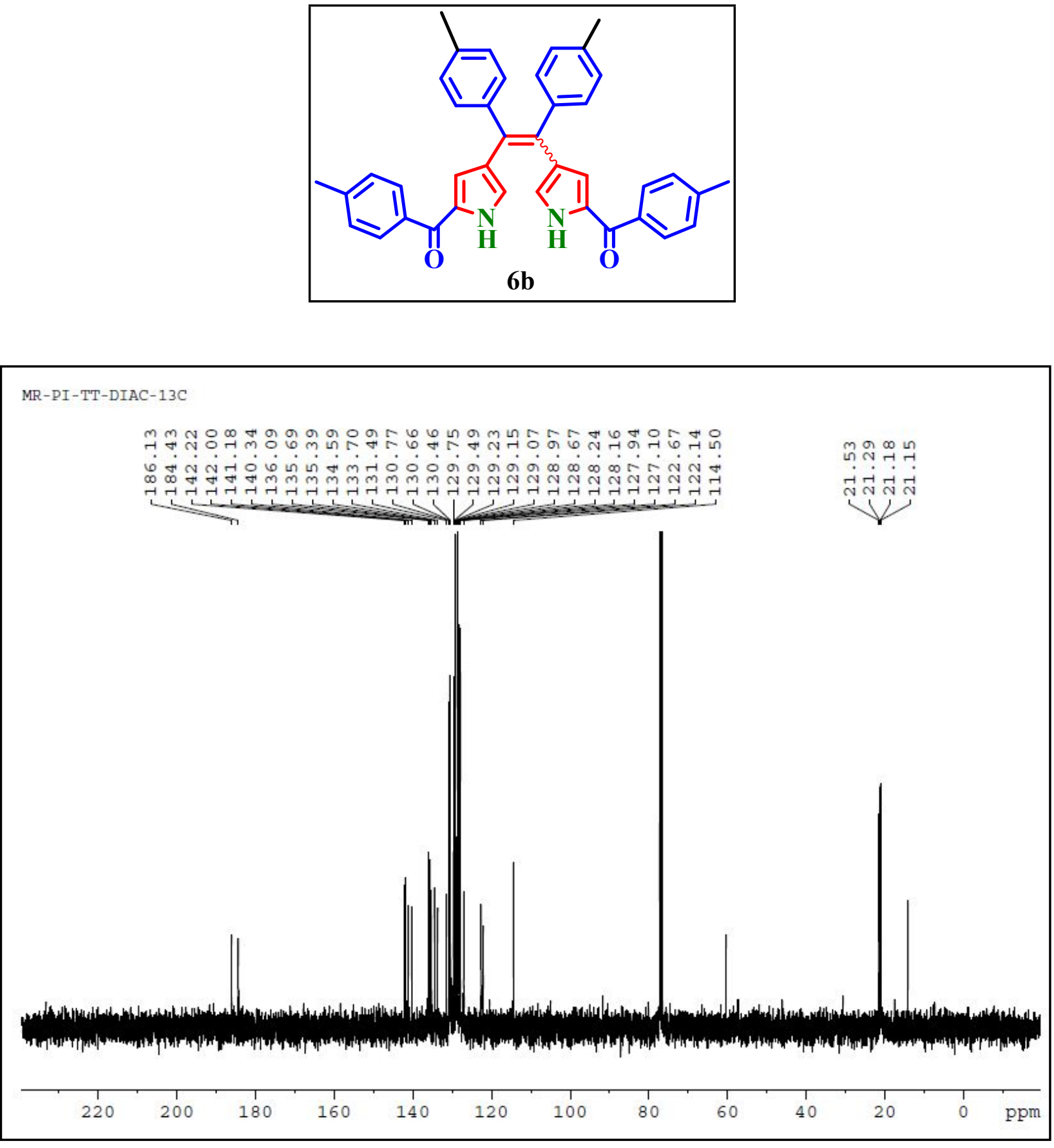

Figure S12. ${ }^{13} \mathrm{C}$ NMR of compound $\mathbf{6 b}$ recorded in $\mathrm{CDCl}_{3}$. 

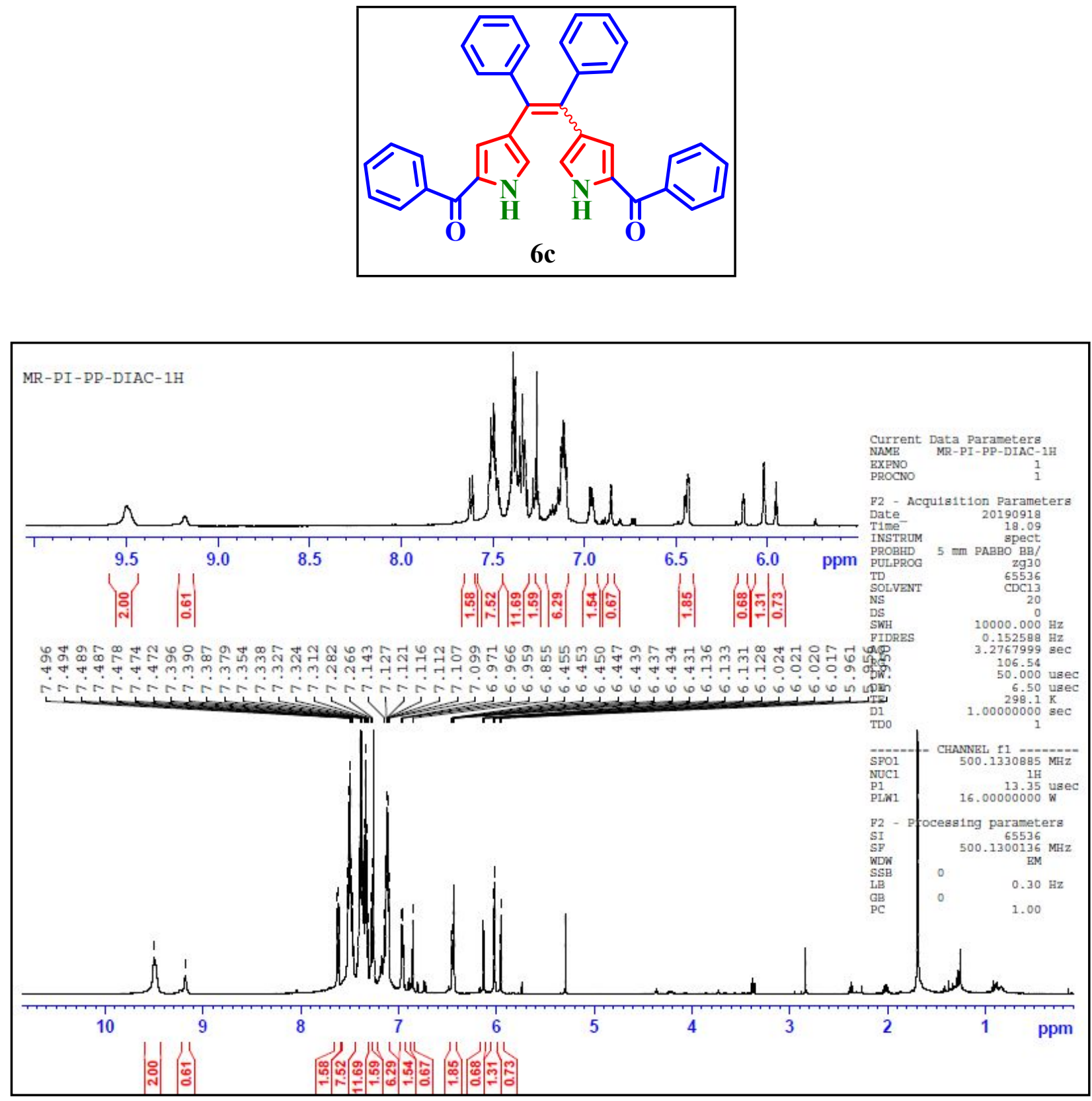

Figure S13. ${ }^{1} \mathrm{H}$ NMR of compound $\mathbf{6 c}$ recorded in $\mathrm{CDCl}_{3}$. 

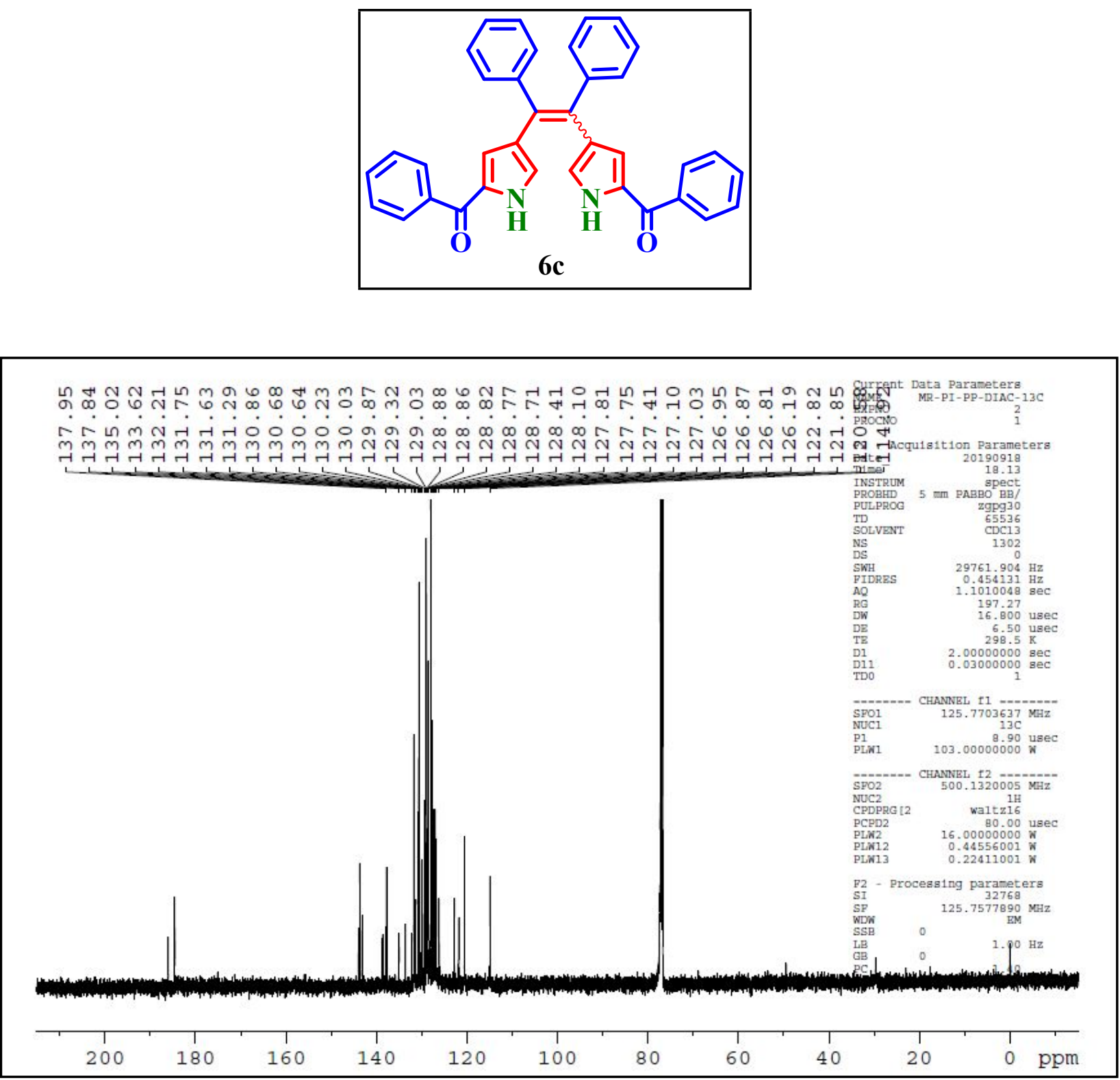

Figure S14. ${ }^{13} \mathrm{C}$ NMR of compound $\mathbf{6 c}$ recorded in $\mathrm{CDCl}_{3}$. 


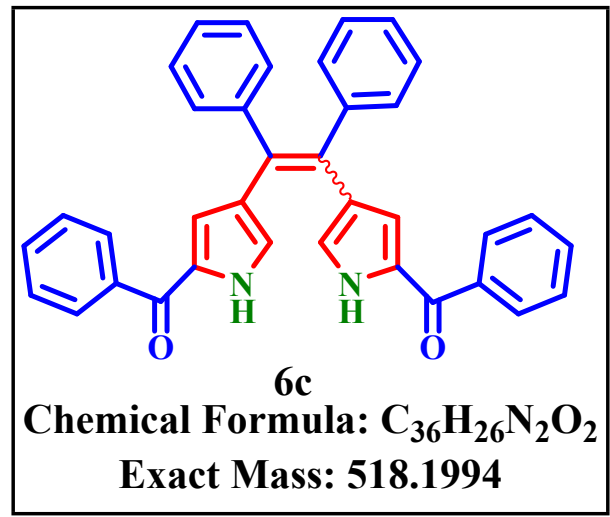

Observed Mass= $541.1887[\mathrm{M}+\mathrm{Na}]^{+}$

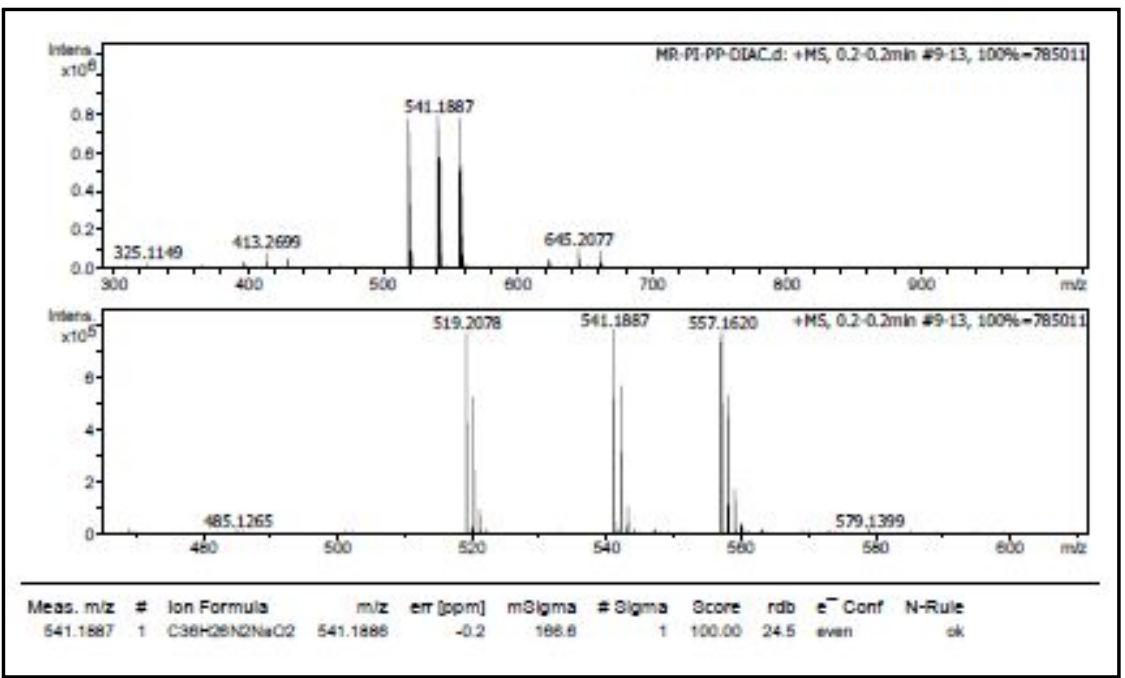

Figure S15. HRMS spectrum of compound $\mathbf{6 c}$. 

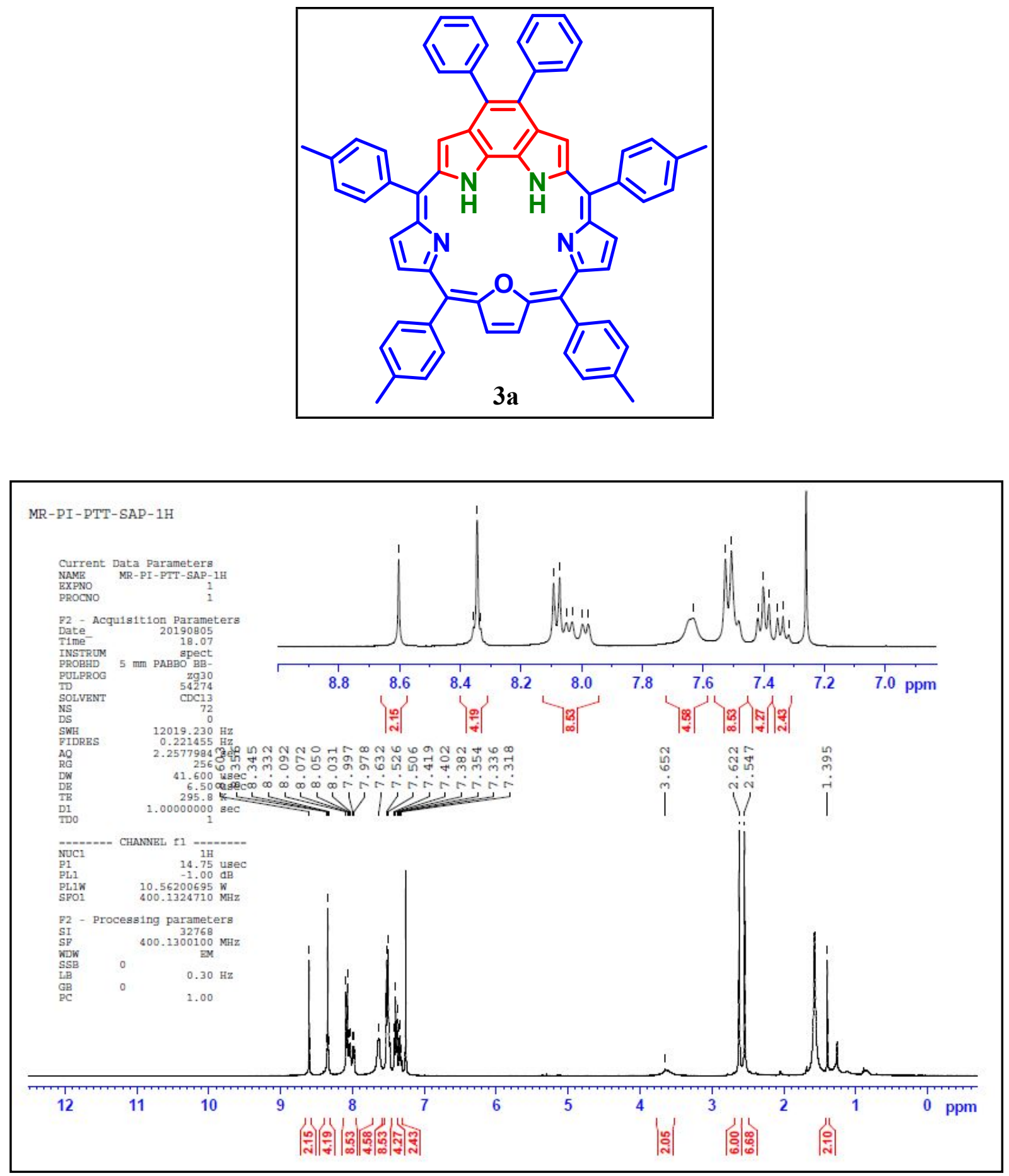

Figure S16. ${ }^{1} \mathrm{H}$ NMR of compound 3a recorded in $\mathrm{CDCl}_{3}$. 

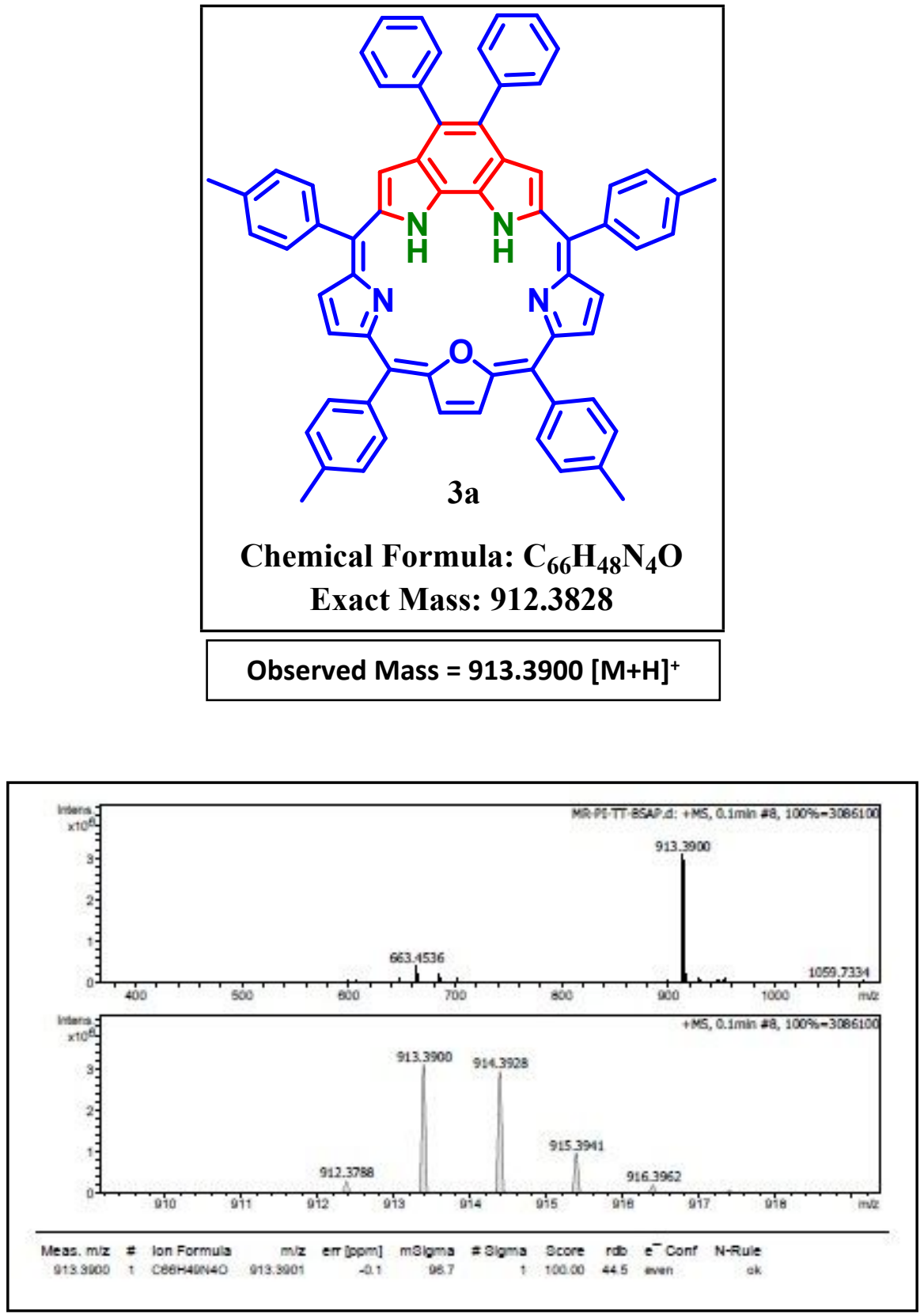

Figure S17. HRMS spectrum of compound 3a. 

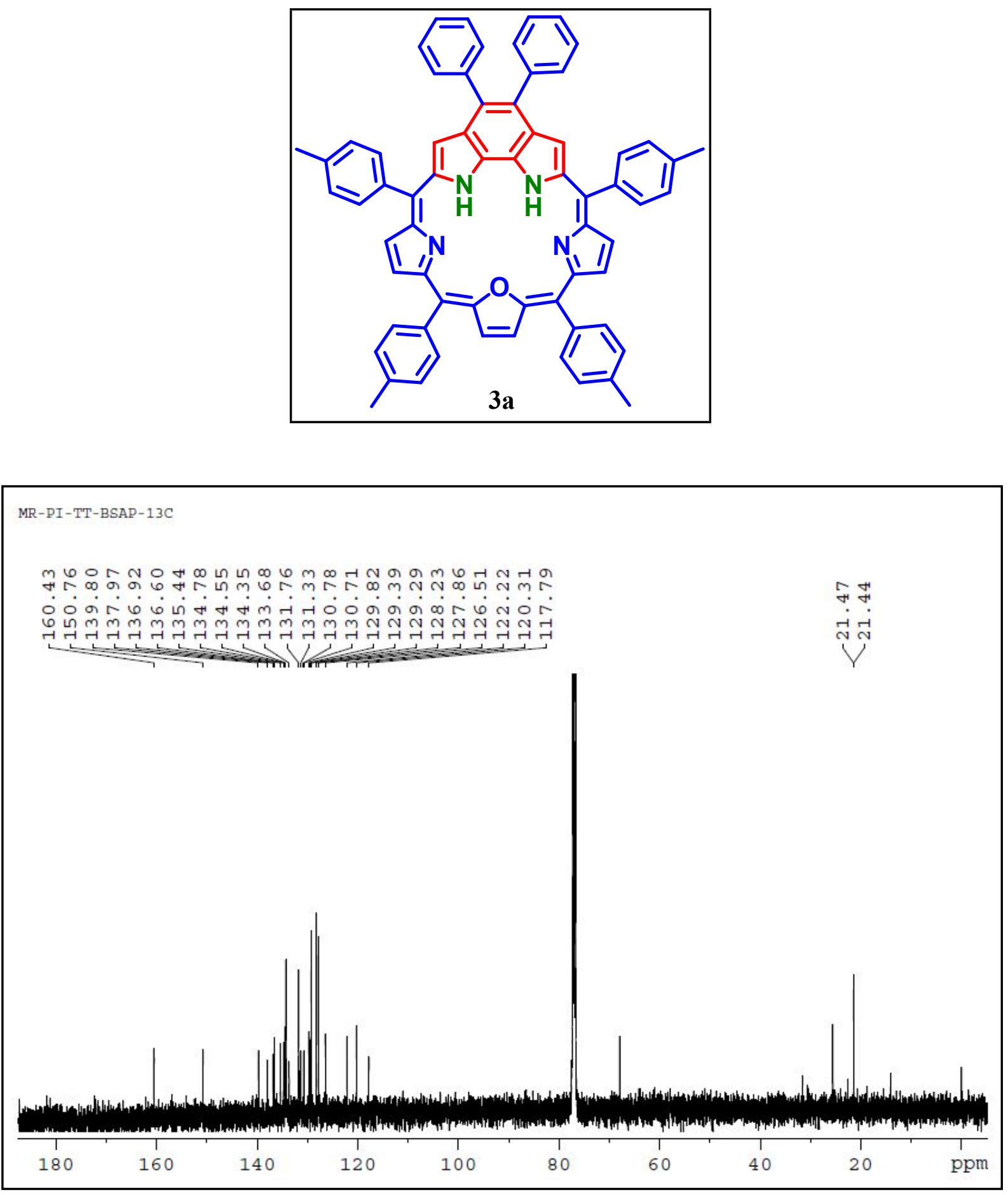

Figure S18. ${ }^{13} \mathrm{C}$ NMR of compound 3a recorded in $\mathrm{CDCl}_{3}$. 


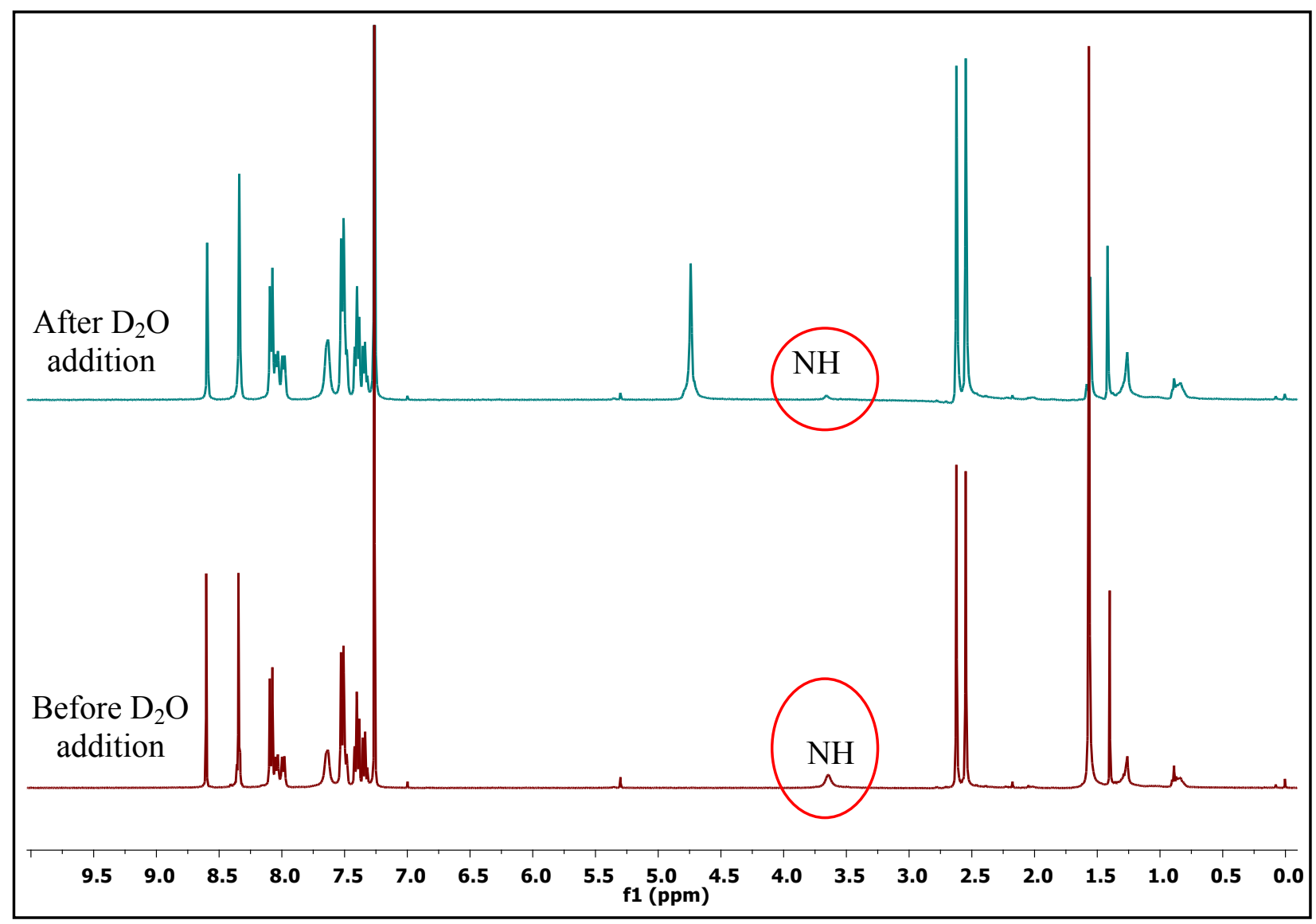

Figure S19. ${ }^{1} \mathrm{H}$ NMR comparison of compound 3a (blue) and after $\mathrm{D}_{2} \mathrm{O}$ addition (brown colour) recorded in $\mathrm{CDCl}_{3}$. 


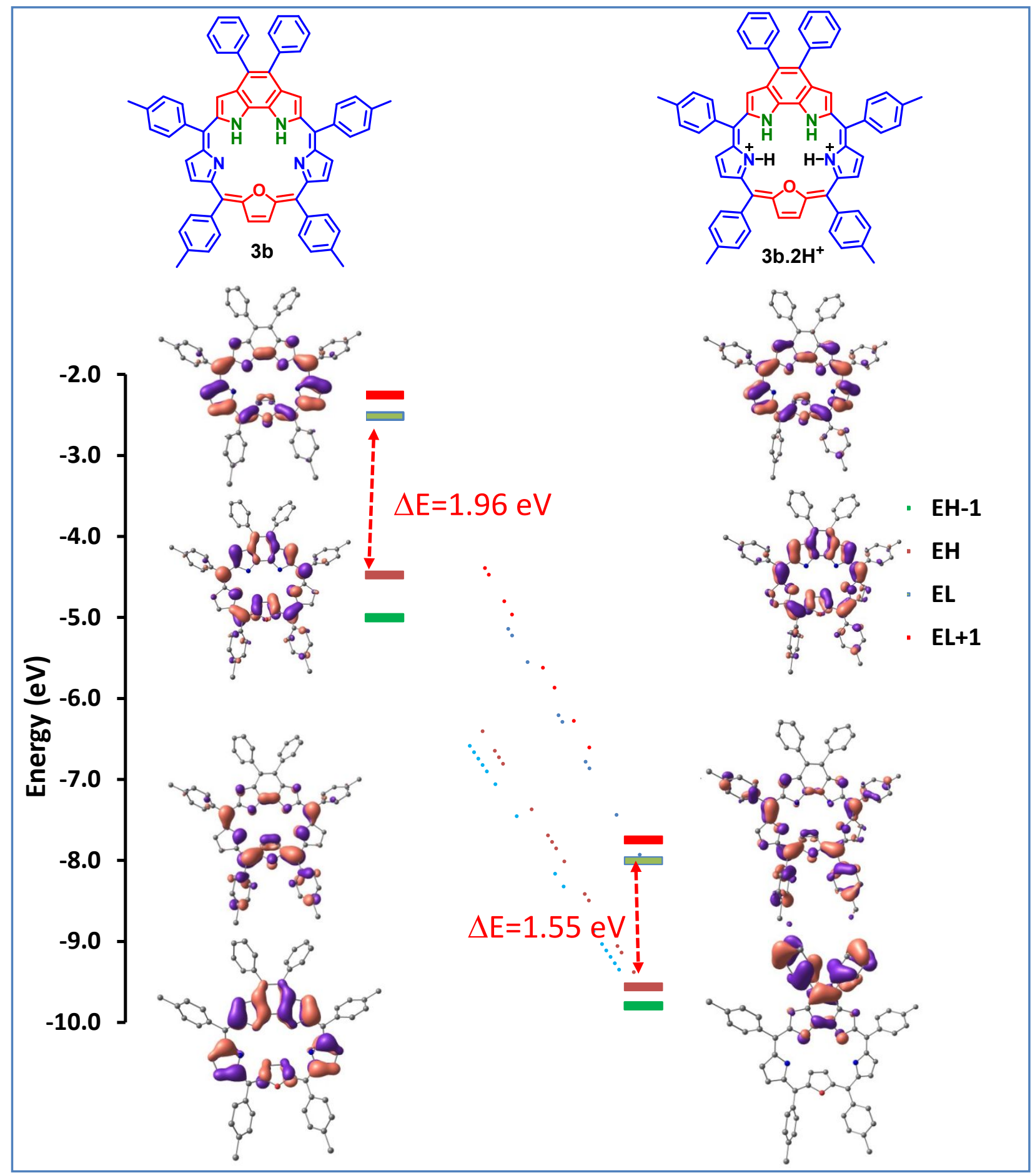

Figure S20. Energy level diagram of compound 3a and [3a.2H $]^{2+}$. 


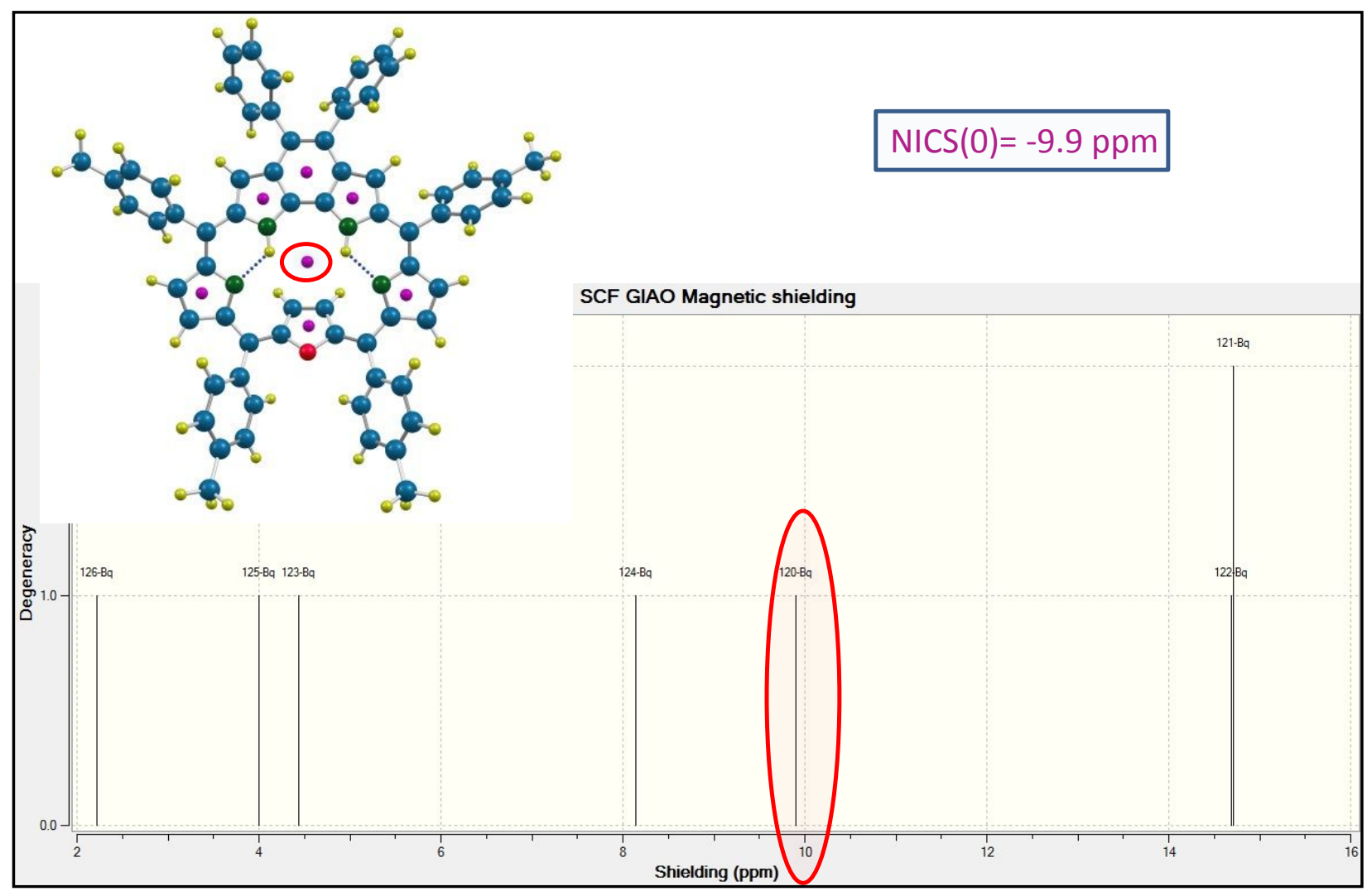

Figure S21. NICS(0) value for macrocycle $3 \mathbf{a}$. 

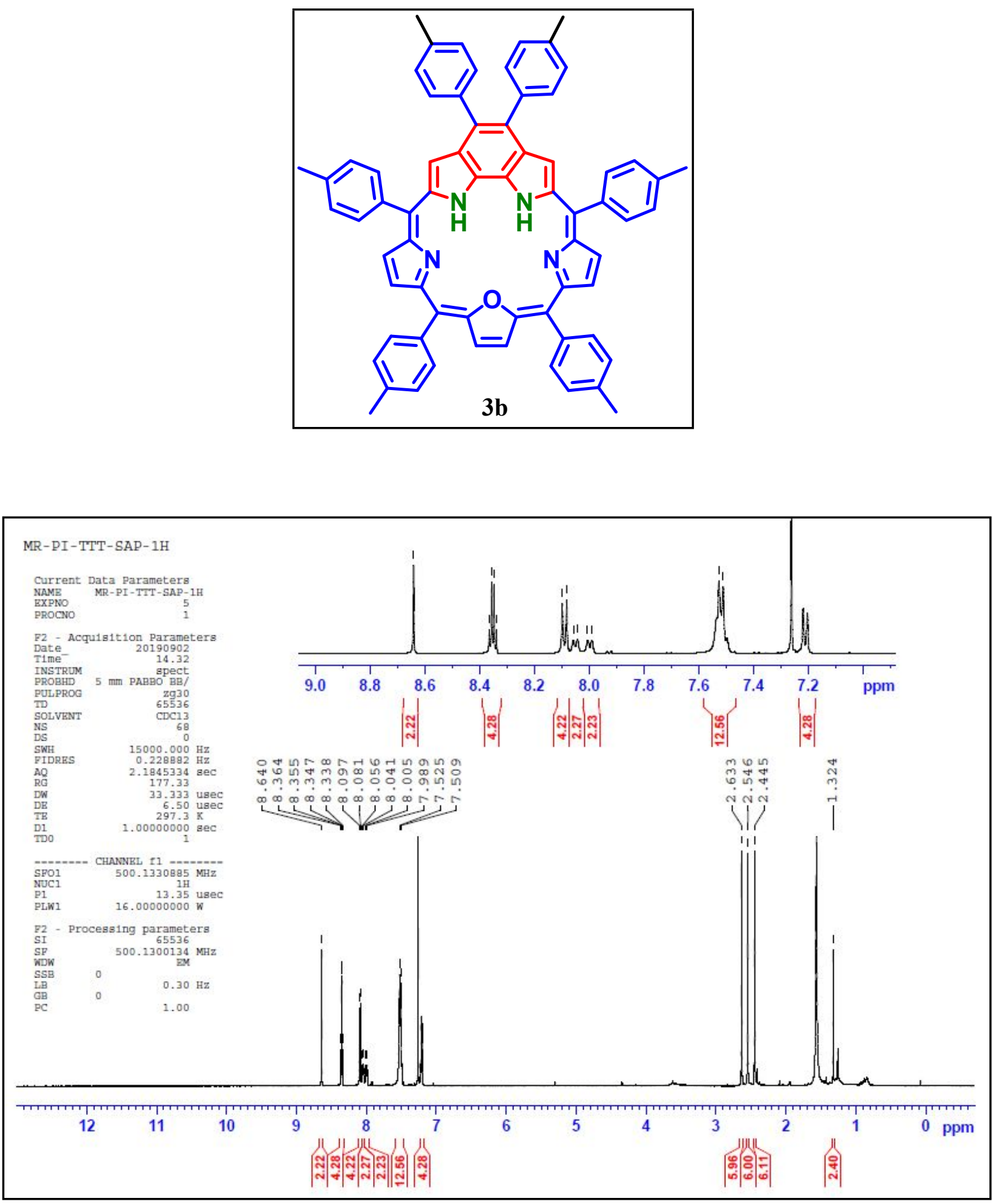

Figure S22. ${ }^{1} \mathrm{H}$ NMR of compound $\mathbf{3 b}$ recorded in $\mathrm{CDCl}_{3}$. 


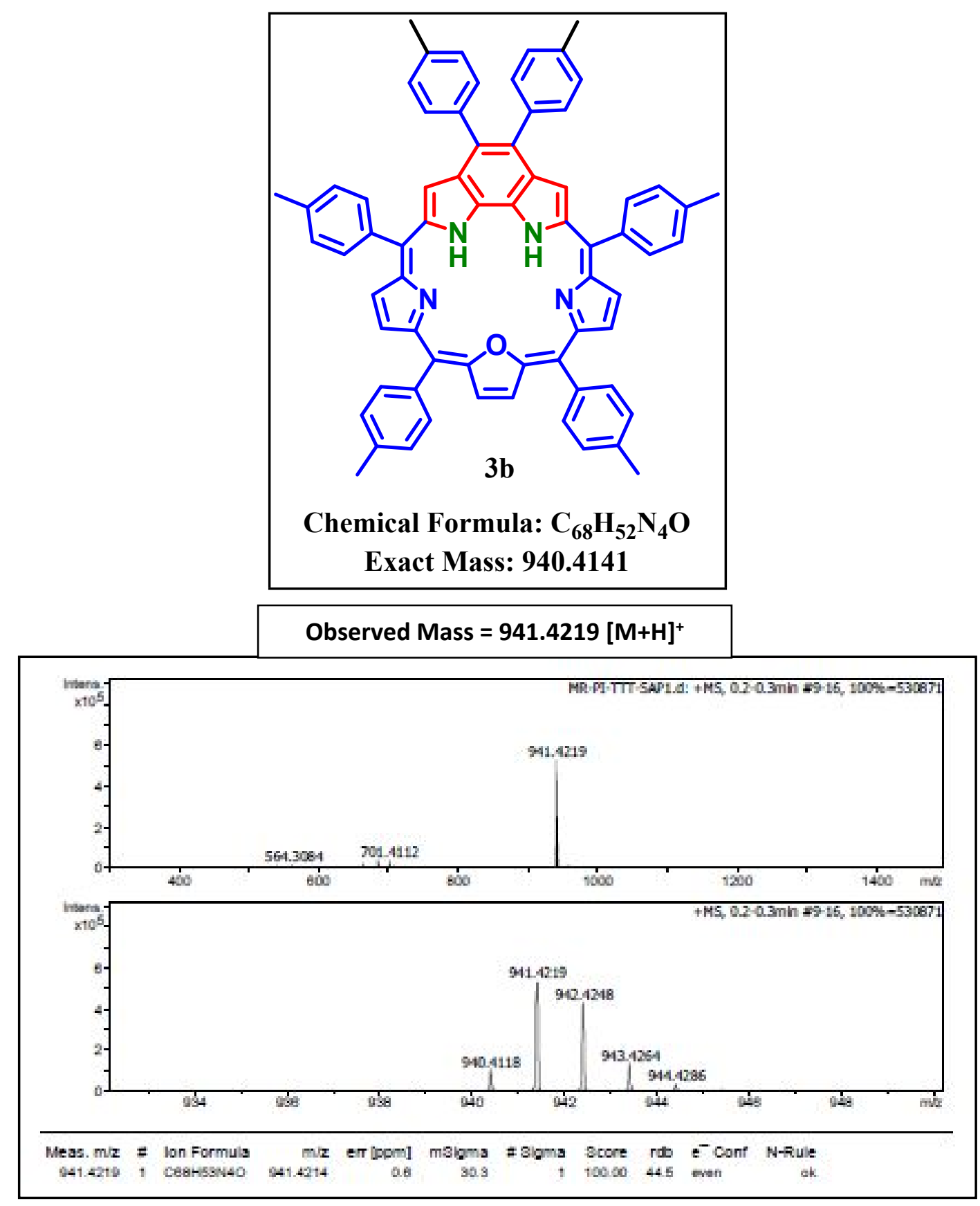

Figure S23. HRMS spectrum of compound $\mathbf{3 b}$. 

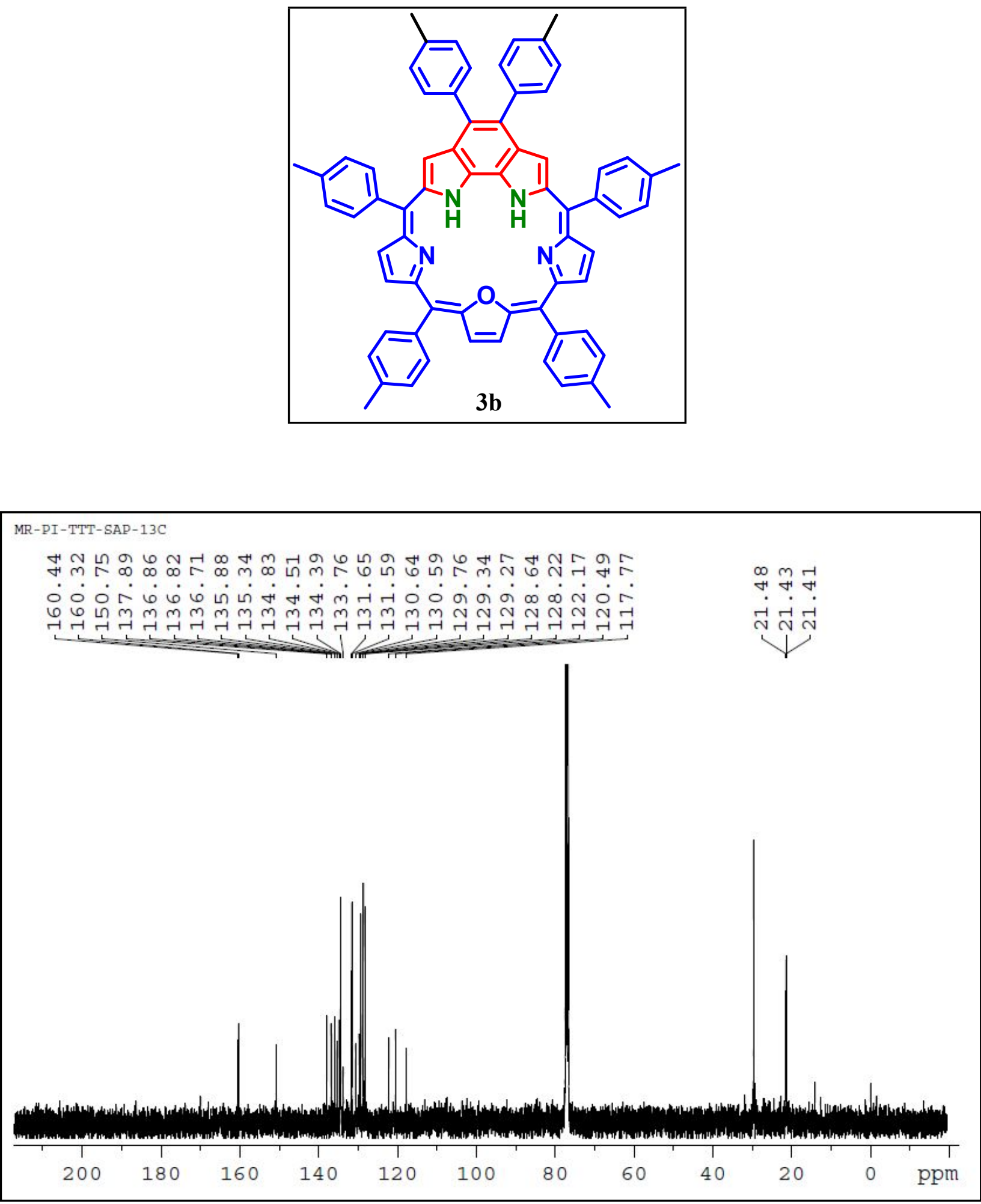

Figure S24. ${ }^{13} \mathrm{C}$ NMR of compound $\mathbf{3 b}$ recorded in $\mathrm{CDCl}_{3}$. 

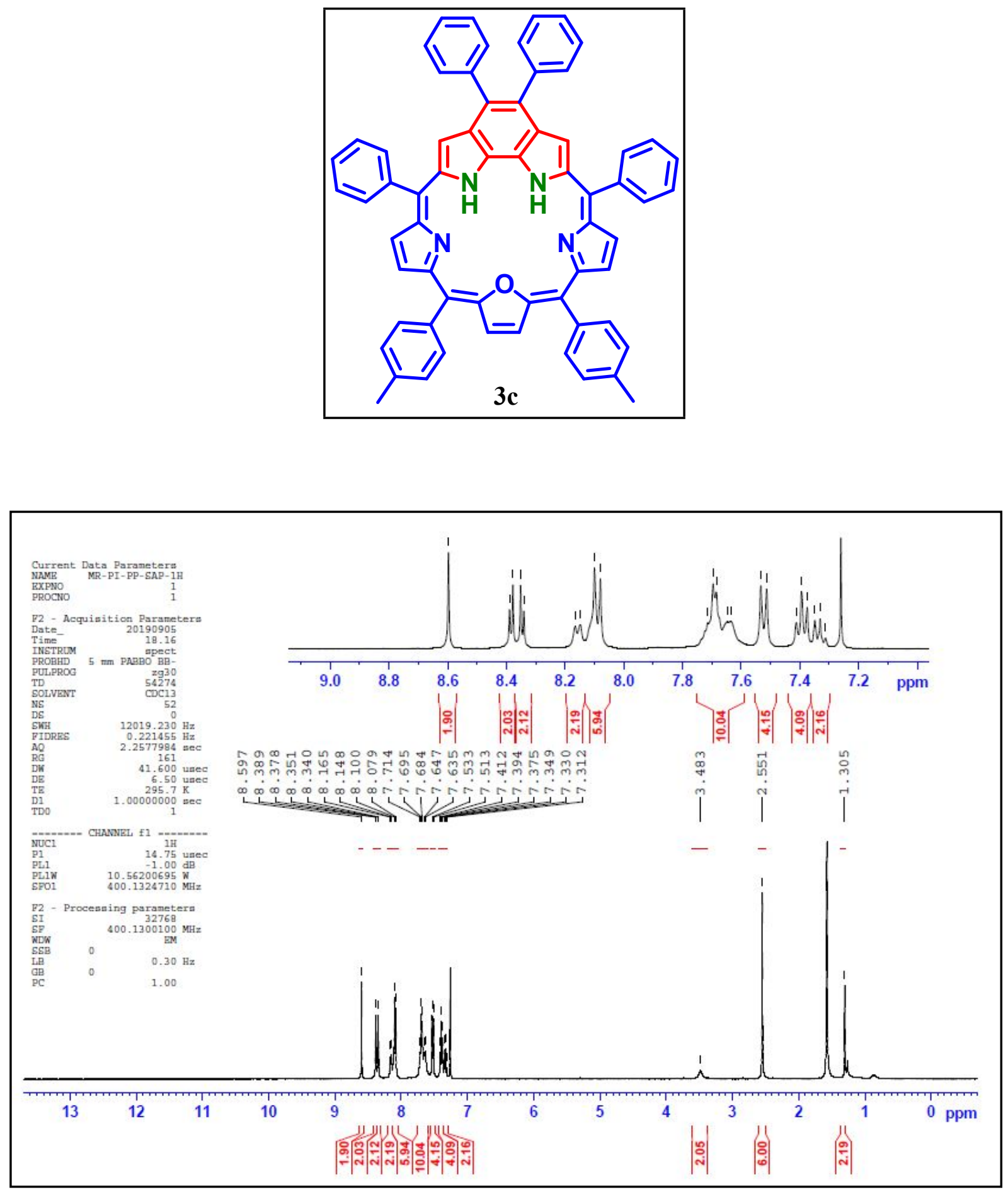

Figure S25. ${ }^{1} \mathrm{H} \mathrm{NMR}$ of compound $\mathbf{3 c}$ recorded in $\mathrm{CDCl}_{3}$. 


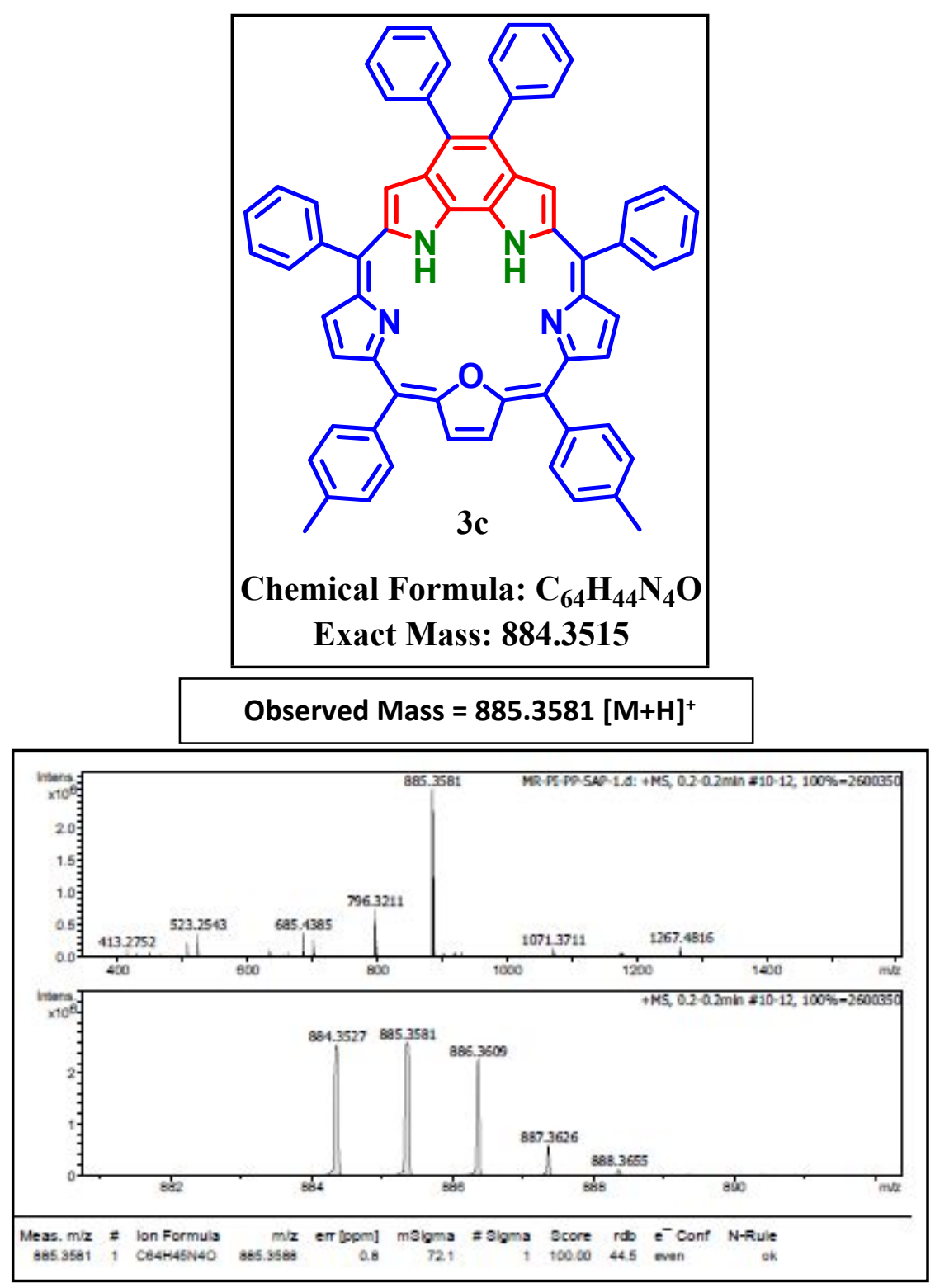

Figure S26. HRMS spectrum of compound 3c. 

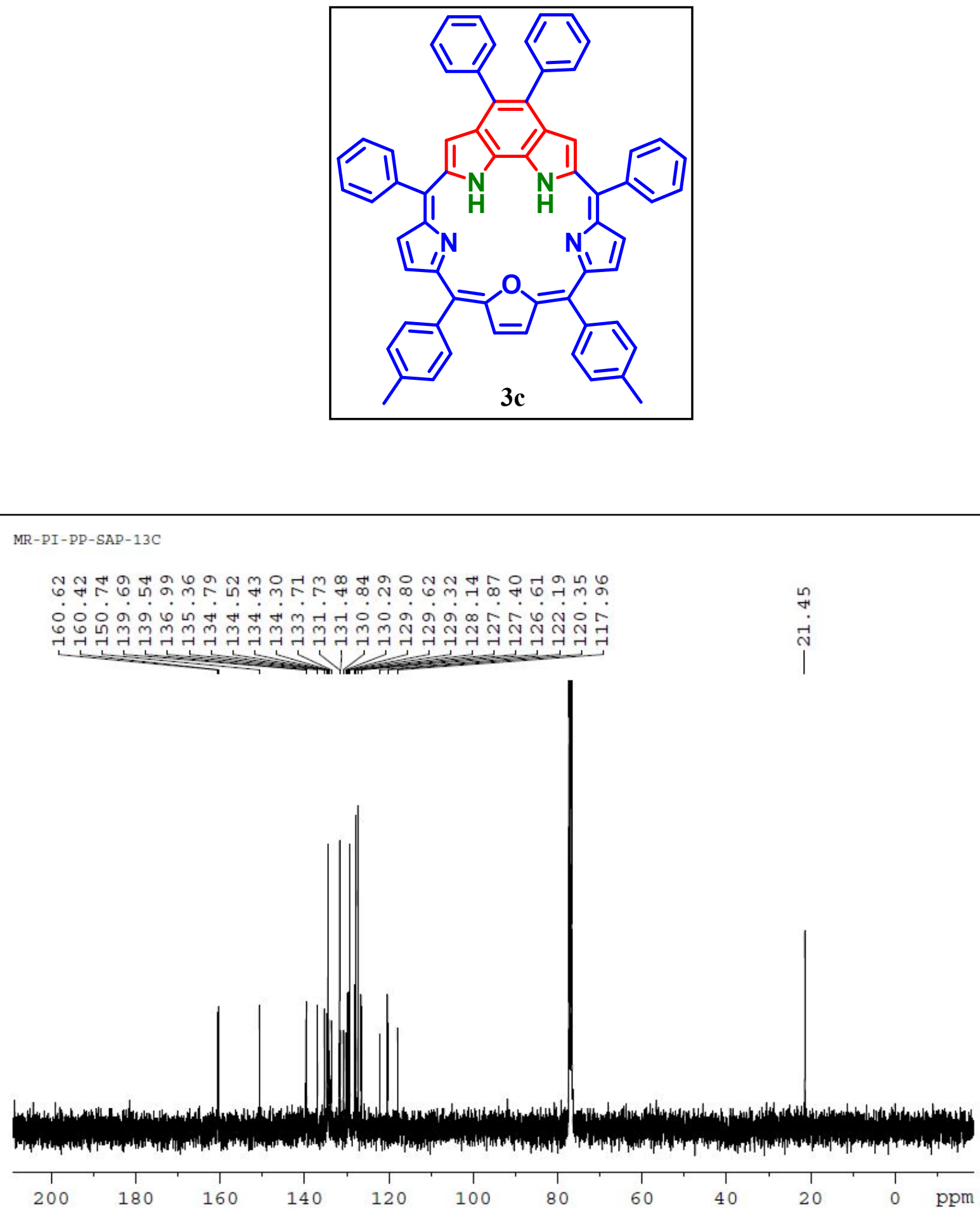

Figure S27. ${ }^{13} \mathrm{C}$ NMR of compound $\mathbf{3 c}$ recorded in $\mathrm{CDCl}_{3}$. 


\begin{tabular}{rrrrrr}
\hline Compound & $\lambda_{\text {abs }} / \mathrm{nm}(\log \varepsilon)$ & $\mathrm{I}$ & $\mathrm{II}$ & $\mathrm{I}$ & $\mathrm{II}$ \\
\cline { 3 - 6 } & & $\mathrm{E}_{\mathrm{ox}}(\mathrm{V})$ & $\mathrm{E}_{\mathrm{ox}}(\mathrm{V})$ & $\mathrm{E}_{\mathrm{red}}(\mathrm{V})$ & $\mathrm{E}_{\mathrm{red}}(\mathrm{V})$ \\
\hline $\mathbf{3 a}$ & $511(4.90), 533(\mathrm{sh}), 722(4.00)$ & 0.77 & 1.11 & -0.73 & -0.93 \\
{$[3 \mathbf{3 a . 2 H}]^{2+}$} & $543(4.96), 804(4.14)$ & - & - & - & - \\
$\mathbf{3 b}$ & $511(4.99), 530(\mathrm{sh}), 726(4.02)$ & 0.78 & 1.12 & -0.74 & -0.90 \\
{$[3 \mathbf{b} .2 \mathrm{H}]^{2+}$} & $545(4.99), 801(4.19)$ & - & - & - & - \\
$\mathbf{3 c}$ & $511(5.02), 529(\mathrm{sh}), 723(4.05)$ & 0.80 & 1.12 & -0.70 & -0.90 \\
{$[\mathbf{3 c . 2 H}]^{2+}$} & $542(5.05), 801(4.19)$ & - & - & - & - \\
\hline
\end{tabular}

Figure S28. Table of absorption and electrochemical data of 3a-3c along with their diprotonated species $[3 \mathbf{a} .2 \mathrm{H}]^{2+}-[\mathbf{3 c} .2 \mathrm{H}]^{2+}$. 
Figure S29. Table of crystal data and data collection parameters for compound 3a.

$\begin{array}{llllll}\text { Atom } & \text { Atom Length/A } & \text { Atom } & \text { Atom Length/A } \\ \text { C100 } & \text { C026 } & 1.707(10) & \text { C60 } & \text { C61 } & 1.400(8) \\ \text { C11 } & \text { C026 } & 1.748(11) & \text { C27 } & \text { C6 } & 1.506(8) \\ \text { O1 } & \text { C18 } & 1.386(7) & \text { C27 } & \text { C28 } & 1.376(9) \\ \text { O1 } & \text { C21 } & 1.396(7) & \text { C27 } & \text { C32 } & 1.371(9) \\ \text { C12 } & \text { C027 } & 1.735(15) & \text { C63 } & \text { C62 } & 1.388(10) \\ \text { C13 } & \text { C027 } & 1.765(14) & \text { C63 } & \text { C64 } & 1.392(9) \\ \text { C14 } & \text { C026 } & 1.714(9) & \text { C62 } & \text { C61 } & 1.382(10) \\ \text { C15 } & \text { C27 } & 1.727(13) & \text { C62 } & \text { C65 } & 1.497(9) \\ \text { N1 } & \text { C5 } & 1.361(7) & \text { C8 } & \text { C7 } & 1.433(8) \\ \text { N1 } & \text { C2 } & 1.393(7) & \text { C6 } & \text { C7 } & 1.367(9) \\ \text { N2 } & \text { C9 } & 1.366(7) & \text { C46 } & \text { C17 } & 1.482(8) \\ \text { N2 } & \text { C11 } & 1.388(7) & \text { C46 } & \text { C47 } & 1.392(10) \\ \text { N3 } & \text { C16 } & 1.340(7) & \text { C46 } & \text { C51 } & 1.400(9) \\ \text { N3 } & \text { C13 } & 1.394(8) & \text { C30 } & \text { C31 } & 1.360(11) \\ \text { N4 } & \text { C23 } & 1.355(8) & \text { C30 } & \text { C29 } & 1.370(11) \\ \text { N4 } & \text { C26 } & 1.355(7) & \text { C21 } & \text { C22 } & 1.387(9) \\ \text { C19 } & \text { C20 } & 1.355(8) & \text { C22 } & \text { C53 } & 1.465(9) \\ \text { C19 } & \text { C18 } & 1.425(9) & \text { C47 } & \text { C48 } & 1.375(9) \\ \text { C20 } & \text { C21 } & 1.408(9) & \text { C25 } & \text { C24 } & 1.330(9) \\ \text { C3 } & \text { C2 } & 1.393(9) & \text { C7 } & \text { C33 } & 1.491(8) \\ \text { C3 } & \text { C4 } & 1.401(8) & \text { C48 } & \text { C49 } & 1.381(11) \\ \text { C18 } & \text { C17 } & 1.365(9) & \text { C44 } & \text { C43 } & 1.401(10) \\ \text { C9 } & \text { C5 } & 1.398(8) & \text { C53 } & \text { C58 } & 1.372(9) \\ \text { C9 } & \text { C8 } & 1.426(8) & \text { C53 } & \text { C54 } & 1.394(10) \\ \text { C5 } & \text { C4 } & 1.418(8) & \text { C31 } & \text { C32 } & 1.388(9) \\ \text { C39 } & \text { C12 } & 1.476(8) & \text { C28 } & \text { C29 } & 1.391(10) \\ \text { C39 } & \text { C44 } & 1.390(10) & \text { C49 } & \text { C50 } & 1.399(12) \\ \text { C39 } & \text { C40 } & 1.389(10) & \text { C49 } & \text { C52 } & 1.504(10) \\ \text { C2 } & \text { C1 } & 1.451(8) & \text { C51 } & \text { C50 } & 1.379(10) \\ \text { C12 } & \text { C11 } & 1.427(8) & \text { C33 } & \text { C34 } & 1.360(10) \\ \text { C12 } & \text { C13 } & 1.388(8) & \text { C33 } & \text { C38 } & 1.374(12) \\ \text { C15 } & \text { C16 } & 1.447(8) & \text { C40 } & \text { C41 } & 1.353(10) \\ \text { C15 } & \text { C14 } & 1.350(9) & \text { C58 } & \text { C57 } & 1.403(9) \\ \text { C16 } & \text { C17 } & 1.439(8) & \text { C42 } & \text { C43 } & 1.395(12) \\ \text { C4 } & \text { C6 } & 1.427(8) & \text { C42 } & \text { C41 } & 1.366(12)\end{array}$




$\begin{array}{llllll}\text { C1 } & \text { C59 } & 1.474(8) & \text { C42 } & \text { C45 } & 1.496(10) \\ \text { C1 } & \text { C26 } & 1.390(8) & \text { C54 } & \text { C55 } & 1.392(10) \\ \text { C59 } & \text { C60 } & 1.413(9) & \text { C34 } & \text { C35 } & 1.379(10) \\ \text { C59 } & \text { C64 } & 1.383(9) & \text { C57 } & \text { C56 } & 1.350(11) \\ \text { C11 } & \text { C10 } & 1.407(8) & \text { C36 } & \text { C35 } & 1.345(12) \\ \text { C10 } & \text { C8 } & 1.412(8) & \text { C36 } & \text { C37 } & 1.384(14) \\ \text { C14 } & \text { C13 } & 1.449(8) & \text { C56 } & \text { C55 } & 1.378(11) \\ \text { C23 } & \text { C22 } & 1.425(8) & \text { C56 } & \text { C024 } & 1.516(11) \\ \text { C23 } & \text { C24 } & 1.447(9) & \text { C38 } & \text { C37 } & 1.375(12) \\ \text { C26 } & \text { C25 } & 1.456(8) & & & \end{array}$


Figure S30. Table of selected bond lengths $[\AA]$ and angles $\left[{ }^{\circ}\right]$ for compound $3 a$.

\begin{tabular}{|c|c|c|c|c|c|c|c|}
\hline & & & & & & & \\
\hline C18 & O1 & $\mathrm{C} 21$ & & $\mathrm{C} 4$ & C6 & $\mathrm{C} 27$ & \\
\hline 5 & N1 & $\mathrm{C} 2$ & & C7 & 6 & $\mathrm{C} 4$ & \\
\hline 9 & J2 & $\mathrm{C} 11$ & & 7 & 6 & & \\
\hline 16 & J3 & $\mathrm{C} 13$ & & C47 & $\mathrm{C} 46$ & C11 & \\
\hline 23 & J4 & $\mathrm{C} 26$ & & C47 & $\mathrm{C} 46$ & C51 & \\
\hline 20 & 19 & $\mathrm{C} 18$ & & C51 & $\mathrm{C} 46$ & $\mathrm{C} 17$ & \\
\hline 19 & 20 & $\mathrm{C} 21$ & & C & $\mathrm{C} 30$ & $\mathrm{C} 29$ & \\
\hline & & 4 & & & $\mathrm{C} 17$ & $\mathrm{C} 16$ & \\
\hline 1 & & C19 & & & 17 & 46 & \\
\hline 17 & & 1 & & & 17 & C46 & \\
\hline 17 & 18 & 19 & & & 64 & C63 & \\
\hline 2 & C9 & $\mathrm{C} 5$ & & 1 & $\mathrm{C} 21$ & $\mathrm{C} 20$ & \\
\hline 2 & 9 & $\mathrm{C} 8$ & & $\mathrm{C} 22$ & $\mathrm{C} 21$ & $\mathrm{O}$ & \\
\hline & 9 & $\mathrm{C} 8$ & & 22 & $\mathrm{C} 21$ & $\mathrm{C} 20$ & \\
\hline 1 & C5 & C9 & & 23 & $\mathrm{C} 22$ & C53 & \\
\hline & & $\mathrm{C} 4$ & & $\mathrm{C} 21$ & $\mathrm{C} 22$ & $\mathrm{C} 23$ & \\
\hline & & 14 & & 21 & $\mathrm{C} 22$ & 53 & \\
\hline 44 & م & $\mathrm{C} 12$ & & & $\mathrm{C} 47$ & $C 46$ & \\
\hline 40 & C39 & $\mathrm{C} 12$ & & & $\mathrm{C} 25$ & C26 & \\
\hline 40 & $\cos$ & $\mathrm{C} 44$ & & $\mathrm{C} 8$ & & 33 & \\
\hline 1 & & $\mathrm{C} 1$ & & 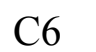 & & & \\
\hline & & $\mathrm{N}$ & & C & & C33 & \\
\hline & $\mathrm{C} 2$ & $\mathrm{C} 1$ & & $\mathrm{C} 25$ & 24 & 23 & \\
\hline 11 & 17 & $\mathrm{C} 3 \mathrm{~S}$ & & C47 & C & C49 & \\
\hline 13 & 12 & $\mathrm{C} 3$ & & C & C44 & C43 & \\
\hline 13 & 12 & $\mathrm{C} 11$ & & C58 & $\mathrm{C} 53$ & $\mathrm{C} 22$ & \\
\hline 14 & C15 & C16 & & C58 & $\mathrm{C} 53$ & C54 & \\
\hline 3 & C16 & $\mathrm{C} 15$ & & C54 & C53 & $\mathrm{C} 22$ & $122.0(6)$ \\
\hline I3 & C16 & $\mathrm{C} 17$ & 119 & $\mathrm{C} 30$ & C31 & C32 & $120.1(7)$ \\
\hline 17 & C16 & C15 & 128. & $\mathrm{C} 27$ & $\mathrm{C} 28$ & $\mathrm{C} 29$ & $120.6(7)$ \\
\hline & $\mathrm{C}^{4}$ & C5 & & C48 & $\mathrm{C} 49$ & C50 & $3(7)$ \\
\hline & $\mathrm{C}^{2}$ & $\mathrm{C}$ & & C 48 & $\mathrm{C} 49$ & C52 & \\
\hline $\mathrm{c} 5$ & $\mathrm{C} 4$ & C6 & $122.6(5)$ & $\mathrm{C} 50$ & $\mathrm{C} 49$ & C52 & $122.2(8$ \\
\hline
\end{tabular}




\begin{tabular}{|c|c|c|c|c|c|c|c|}
\hline & & & & & & C46 & \\
\hline & & & & & & & \\
\hline & 1 & & & 34 & 33 & & \\
\hline & 9 & 1 & & 38 & 33 & & \\
\hline & 59 & & & 27 & C32 & & \\
\hline & 59 & & & 30 & C29 & 28 & \\
\hline & 1 & & & C41 & 40 & & \\
\hline & 1 & & & C53 & & & \\
\hline & & & & 51 & C. & & \\
\hline & 10 & & & 43 & C42 & +5 & \\
\hline & 14 & ( & & 41 & C42 & & \\
\hline & 23 & & & 41 & C42 & & \\
\hline & 23 & T & & 55 & 54 & 33 & \\
\hline & 23 & & & 42 & 43 & & \\
\hline & 26 & & & 33 & 34 & & \\
\hline & 26 & & & 56 & & & \\
\hline & 26 & & & 35 & $\mathrm{C} 36$ & 37 & \\
\hline & 60 & & & 57 & C56 & & \\
\hline & 27 & & & 57 & & & \\
\hline & 27 & & & 55 & & & \\
\hline & $\mathrm{C} 27$ & $\mathrm{C} 2$ & & $\mathrm{C} 36$ & C35 & & \\
\hline & 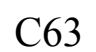 & $G c$ & & $\mathrm{C} 40$ & C & & \\
\hline & & & & 56 & . & & \\
\hline & C & & & 33 & C & 37 & \\
\hline & C & $\mathrm{C}$ & & 30 & & & \\
\hline & ar & & & 100 & & & \\
\hline & a & C6 & & 0 & & & \\
\hline & C6 & $c$ & & 14 & O & & \\
\hline & $\mathrm{C} 8$ & C7 & & 12 & $\mathrm{C} 027$ & $\mathrm{Cl} 3$ & (7) \\
\hline & $\mathrm{C} 8$ & & & $\mathrm{C} 15$ & 27 & 12 & (7) \\
\hline & C8 & C7 & & $\mathrm{Cl} 5$ & $\mathrm{C} 027$ & $\mathrm{Cl} 3$ & $107.4(9)$ \\
\hline & 661 & $\mathrm{C} 60$ & $121.8(6)$ & & & & \\
\hline
\end{tabular}




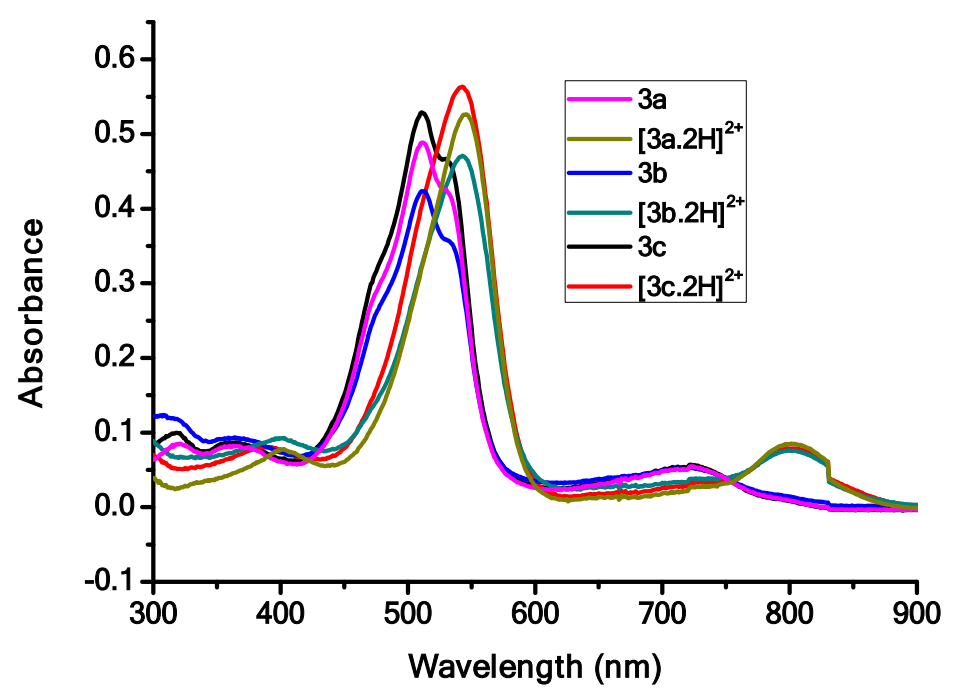

Figure S31: Comparison of absorption spectra of 3a-3c and their diprotonated compounds $[3 \mathbf{a} .2 \mathrm{H}]^{2+}-[3 \mathbf{c} .2 \mathbf{H}]^{2+}$ recorded in $\mathrm{CHCl}_{3}$.
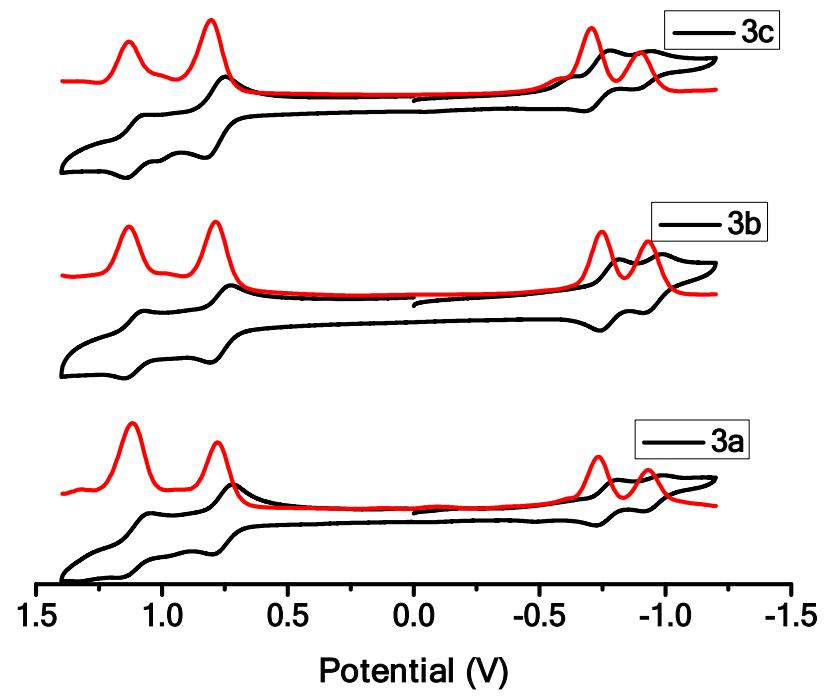

Figure S32: Comparison of cyclic voltammogram of compounds 3a-3c (black line) along with differential pulse voltammogram (red line), recorded in $\mathrm{CH}_{2} \mathrm{Cl}_{2}$ containing $0.1 \mathrm{M}$ TBAP as the supporting electrolyte and a saturated calomel electrode (SCE) as the reference electrode at scan rates of $50 \mathrm{mV} \mathrm{s}^{-1}$. 
Figure S33: Cartesian coordinates of the S0 optimized structures of the compounds 3a.

\# Sum of imaginary frequencies $=0$; \# Total Energy (hartree) $=-2838.18319911$

\begin{tabular}{|c|c|c|c|c|c|c|c|}
\hline Atoms & $x$ & $Y$ & $Z$ & Atoms & $x$ & $Y$ & $Z$ \\
\hline 0 & 4.22509 & -0.03354 & -0.62814 & C & 7.6143 & -2.54818 & -0.36942 \\
\hline $\mathrm{N}$ & -0.82938 & 1.64218 & -0.15048 & $\mathrm{H}$ & 8.4636 & -2.16047 & -0.92808 \\
\hline $\mathrm{H}$ & 0.187 & 1.53684 & -0.18668 & C & -5.58129 & 1.5445 & 0.23392 \\
\hline $\mathrm{N}$ & 1.52952 & 2.96868 & -0.30586 & C & -2.8159 & -2.74641 & 0.0036 \\
\hline $\mathrm{N}$ & -0.85146 & -1.6453 & -0.11078 & $\mathrm{H}$ & -3.5346 & -3.55012 & 0.036 \\
\hline $\mathrm{H}$ & 0.16445 & -1.5551 & -0.18201 & C & -4.34186 & 0.71652 & 0.15807 \\
\hline $\mathrm{N}$ & 1.47212 & -3.00253 & -0.41348 & C & -2.99674 & 6.95767 & -1.01728 \\
\hline C & -1.34048 & 5.41774 & -0.11625 & $\mathrm{H}$ & -3.75065 & 7.18066 & -1.76929 \\
\hline C & 0.78978 & 4.12628 & -0.27901 & C & -7.72398 & 2.23955 & -0.68706 \\
\hline C & 2.82137 & 3.33032 & -0.42542 & $\mathrm{H}$ & -8.4837 & 2.14762 & -1.45903 \\
\hline C & 5.24822 & 2.66516 & -0.08286 & C & -4.35519 & -0.66669 & 0.11961 \\
\hline C & 3.51149 & 1.09115 & -0.98326 & C & -2.71546 & 7.90326 & -0.02106 \\
\hline C & 2.37728 & -0.69102 & -1.76416 & C & -3.09866 & -1.3721 & 0.02028 \\
\hline $\mathrm{H}$ & 1.67476 & -1.34399 & -2.25671 & C & -6.57966 & 1.44546 & -0.7482 \\
\hline C & 1.67592 & 5.27782 & -0.39528 & $\mathrm{H}$ & -6.45297 & 0.74033 & -1.56401 \\
\hline $\mathrm{H}$ & 1.3704 & 6.31422 & -0.439 & C & -5.7621 & 2.46866 & 1.27643 \\
\hline C & 7.89867 & 3.28746 & 0.75037 & $\mathrm{H}$ & -5.001 & 2.55581 & 2.04715 \\
\hline C & -0.6087 & 4.12192 & -0.16925 & C & 7.84054 & -3.35796 & 0.75282 \\
\hline C & 5.48939 & 3.53473 & 0.99729 & C & -1.32654 & -6.37726 & -1.05428 \\
\hline $\mathrm{H}$ & 4.64811 & 3.95896 & 1.53626 & $\mathrm{H}$ & -0.72677 & -6.16837 & -1.9354 \\
\hline C & 2.38811 & 0.67954 & -1.7464 & C & 6.72193 & -3.84892 & 1.4377 \\
\hline $\mathrm{H}$ & 1.69713 & 1.3548 & -2.22545 & $\mathrm{H}$ & 6.86504 & -4.47455 & 2.31619 \\
\hline C & -1.37628 & 2.91785 & -0.10401 & C & -2.01843 & -7.58331 & -0.95725 \\
\hline C & -1.8257 & 0.70573 & -0.0445 & $\mathrm{H}$ & -1.94206 & -8.30728 & -1.76565 \\
\hline C & 5.19885 & -2.73217 & -0.1031 & C & 9.3054 & 3.59261 & 1.20656 \\
\hline C & 6.36848 & 2.10989 & -0.73253 & $\mathrm{H}$ & 9.35016 & 4.53027 & 1.77024 \\
\hline $\mathrm{H}$ & 6.21842 & 1.44524 & -1.57734 & $\mathrm{H}$ & 9.99386 & 3.67418 & 0.35786 \\
\hline C & 6.78756 & 3.83989 & 1.3988 & $\mathrm{H}$ & 9.6926 & 2.79949 & 1.86058 \\
\hline $\mathrm{H}$ & 6.93954 & 4.51277 & 2.24002 & C & -5.94949 & -2.3859 & -0.79389 \\
\hline C & -3.07357 & 1.40247 & 0.07124 & $\mathrm{H}$ & -5.30628 & -2.4798 & -1.66471 \\
\hline C & 1.61073 & -5.30897 & -0.27676 & C & -2.21801 & -5.70199 & 1.07645 \\
\hline $\mathrm{H}$ & 1.30482 & -6.34203 & -0.18574 & $\mathrm{H}$ & -2.29395 & -4.97692 & 1.88143 \\
\hline C & 0.7268 & -4.15009 & -0.29961 & C & -6.45467 & -1.36579 & 1.33174 \\
\hline C & -1.05944 & 6.36337 & 0.8824 & $\mathrm{H}$ & -6.20103 & -0.66973 & 2.12549 \\
\hline $\mathrm{H}$ & -0.31611 & 6.13057 & 1.63918 & C & -2.81678 & -7.87549 & 0.15551 \\
\hline C & 2.94405 & 4.78209 & -0.4619 & C & -6.90832 & 3.26204 & 1.33947 \\
\hline $\mathrm{H}$ & 3.86602 & 5.33555 & -0.58115 & $\mathrm{H}$ & -7.03114 & 3.96563 & 2.15893 \\
\hline C & -2.32591 & 5.73861 & -1.06623 & C & -7.10826 & -3.15748 & -0.7006 \\
\hline $\mathrm{H}$ & -2.5545 & 5.02902 & -1.85596 & $\mathrm{H}$ & -7.35849 & -3.8514 & -1.49905 \\
\hline C & 6.32532 & -2.2385 & -0.78859 & C & -7.8935 & 3.15098 & 0.35698 \\
\hline $\mathrm{H}$ & 6.18232 & -1.62309 & -1.67158 & $\mathrm{H}$ & -8.78592 & 3.76952 & 0.40435 \\
\hline C & -1.83703 & -0.69402 & -0.05209 & C & -2.90399 & -6.91064 & 1.1683 \\
\hline C & 3.87656 & 2.35753 & -0.5249 & $\mathrm{H}$ & -3.51629 & -7.10919 & 2.04532 \\
\hline
\end{tabular}




\begin{tabular}{lccccccc}
$\mathrm{C}$ & 5.42793 & -3.54183 & 1.02476 & $\mathrm{C}$ & -7.9437 & -3.03689 & 0.41102 \\
$\mathrm{H}$ & 4.58123 & -3.91709 & 1.59098 & $\mathrm{H}$ & -8.84592 & -3.63827 & 0.48558 \\
$\mathrm{C}$ & 7.66065 & 2.41751 & -0.3242 & $\mathrm{C}$ & 9.2426 & -3.66431 & 1.22273 \\
$\mathrm{H}$ & 8.50438 & 1.98022 & -0.85399 & $\mathrm{H}$ & 9.94407 & -3.72038 & 0.38308 \\
$\mathrm{C}$ & -2.77194 & 2.77232 & 0.03653 & $\mathrm{H}$ & 9.28605 & -4.61496 & 1.76477 \\
$\mathrm{H}$ & -3.47689 & 3.5858 & 0.10498 & $\mathrm{H}$ & 9.61338 & -2.88521 & 1.90273 \\
$\mathrm{C}$ & 3.83186 & -2.41515 & -0.56016 & $\mathrm{C}$ & -7.61137 & -2.1385 & 1.42691 \\
$\mathrm{C}$ & -5.60632 & -1.47442 & 0.21859 & $\mathrm{H}$ & -8.25329 & -2.03944 & 2.2985 \\
$\mathrm{C}$ & 3.4924 & -1.13875 & -1.00977 & $\mathrm{C}$ & -3.54217 & -9.19528 & 0.27349 \\
$\mathrm{C}$ & -1.73776 & 7.58088 & 0.92742 & $\mathrm{H}$ & -3.71557 & -9.64644 & -0.70904 \\
$\mathrm{H}$ & -1.50699 & 8.28988 & 1.71948 & $\mathrm{H}$ & -2.96283 & -9.91625 & 0.86594 \\
$\mathrm{C}$ & 2.76643 & -3.37476 & -0.46145 & $\mathrm{H}$ & -4.51247 & -9.07734 & 0.76825 \\
$\mathrm{C}$ & 2.88155 & -4.82511 & -0.37092 & $\mathrm{C}$ & -3.43198 & 9.23267 & 0.01157 \\
$\mathrm{H}$ & 3.8032 & -5.39054 & -0.40262 & $\mathrm{H}$ & -3.3298 & 9.71903 & 0.98709 \\
$\mathrm{C}$ & -1.41266 & -5.41164 & -0.03802 & $\mathrm{H}$ & -3.02788 & 9.92141 & -0.74236 \\
$\mathrm{C}$ & -1.41759 & -2.91241 & -0.07624 & $\mathrm{H}$ & -4.5014 & 9.1177 & -0.19872 \\
$\mathrm{C}$ & -0.66743 & -4.12731 & -0.14256 & & & & \\
\hline
\end{tabular}


Figure S34: Cartesian coordinates of the S0 optimized structures of the compounds $[\mathbf{3 a} . \mathbf{2 H}]^{2+}$.

\# Sum of imaginary frequencies $=0$; \# Total Energy (hartree) $=-2838.93237255$

\begin{tabular}{|c|c|c|c|c|c|c|c|}
\hline Atoms & $x$ & $Y$ & $Z$ & Atoms & $x$ & $Y$ & $Z$ \\
\hline 0 & 4.17884 & -0.13557 & -0.46637 & $\mathrm{H}$ & 8.41418 & -2.43573 & -1.08648 \\
\hline $\mathrm{N}$ & -0.8152 & 1.60479 & -0.09515 & C & -5.58624 & 1.65218 & 0.39473 \\
\hline $\mathrm{H}$ & -0.0513 & 1.39692 & -0.72695 & $C$ & -2.92102 & -2.69431 & -0.15103 \\
\hline $\mathrm{N}$ & 1.6515 & 2.97947 & -0.11276 & $\mathrm{H}$ & -3.6537 & -3.48138 & -0.24484 \\
\hline$N$ & -0.91147 & -1.61913 & -0.09947 & C & -4.38093 & 0.79249 & 0.22626 \\
\hline $\mathrm{H}$ & -0.10792 & -1.48507 & 0.50649 & C & -2.96326 & 6.89881 & -1.02609 \\
\hline $\mathrm{N}$ & 1.40878 & -3.10851 & -0.58349 & $\mathrm{H}$ & -3.77416 & 7.074 & -1.72812 \\
\hline C & -1.26036 & 5.41052 & -0.13195 & $C$ & -7.70814 & 2.50428 & -0.42165 \\
\hline C & 0.84609 & 4.10359 & -0.22832 & $\mathrm{H}$ & -8.48265 & 2.51355 & -1.18298 \\
\hline C & 2.98006 & 3.29931 & -0.33663 & C & -4.43547 & -0.58582 & 0.04155 \\
\hline C & 5.36304 & 2.58392 & 0.02445 & $\mathrm{C}$ & -2.57047 & 7.92826 & -0.15548 \\
\hline C & 3.56976 & 1.02734 & -0.86094 & C & -3.19213 & -1.31335 & -0.07272 \\
\hline C & 2.51171 & -0.6767 & -1.88699 & $C$ & -6.59957 & 1.67394 & -0.57665 \\
\hline $\mathrm{H}$ & 1.91247 & -1.25461 & -2.5785 & $\mathrm{H}$ & -6.51599 & 1.04074 & -1.45443 \\
\hline C & 1.72399 & 5.1726 & -0.57632 & $C$ & -5.70844 & 2.482 & 1.52214 \\
\hline $\mathrm{H}$ & 1.40024 & 6.17062 & -0.8333 & $\mathrm{H}$ & -4.94007 & 2.46299 & 2.29119 \\
\hline C & 8.03006 & 3.2232 & 0.76514 & $C$ & 7.79651 & -3.51157 & 0.67711 \\
\hline C & -0.56724 & 4.10444 & -0.118 & $C$ & -1.36547 & -6.45447 & -0.90809 \\
\hline C & 5.63504 & 3.59353 & 0.97499 & $\mathrm{H}$ & -0.6879 & -6.32902 & -1.74776 \\
\hline $\mathrm{H}$ & 4.81356 & 4.11463 & 1.4565 & C & 6.68014 & -3.92358 & 1.42277 \\
\hline C & 2.54383 & 0.70351 & -1.77191 & $\mathrm{H}$ & 6.8293 & -4.49922 & 2.33251 \\
\hline $\mathrm{H}$ & 1.96968 & 1.42143 & -2.34094 & C & -2.07067 & -7.64438 & -0.76741 \\
\hline C & -1.33948 & 2.91594 & -0.02083 & $\mathrm{H}$ & -1.91886 & -8.4406 & -1.49127 \\
\hline C & -1.8786 & 0.71451 & -0.00898 & C & 9.44692 & 3.54828 & 1.15631 \\
\hline C & 5.16101 & -2.84683 & -0.14257 & $\mathrm{H}$ & 9.50008 & 4.44727 & 1.77582 \\
\hline C & 6.46143 & 1.8909 & -0.53749 & $\mathrm{H}$ & 10.07567 & 3.70328 & 0.2721 \\
\hline $\mathrm{H}$ & 6.29079 & 1.1221 & -1.28324 & $\mathrm{H}$ & 9.89354 & 2.72243 & 1.72481 \\
\hline C & 6.94084 & 3.90264 & 1.33213 & C & -6.02549 & -2.17992 & -1.06837 \\
\hline $\mathrm{H}$ & 7.12215 & 4.6768 & 2.07241 & $\mathrm{H}$ & -5.36597 & -2.20256 & -1.93257 \\
\hline C & -3.09079 & 1.44257 & 0.17625 & C & -2.48421 & -5.57236 & 1.04533 \\
\hline C & 1.4778 & -5.29534 & -0.09725 & $\mathrm{H}$ & -2.63353 & -4.78154 & 1.77437 \\
\hline $\mathrm{H}$ & 1.15991 & -6.29423 & 0.16216 & $\mathrm{C}$ & -6.57961 & -1.33509 & 1.12935 \\
\hline C & 0.59214 & -4.19001 & -0.30923 & $\mathrm{H}$ & -6.3421 & -0.71536 & 1.98848 \\
\hline C & -0.85861 & 6.44367 & 0.73764 & $C$ & -2.9836 & -7.83139 & 0.28271 \\
\hline $\mathrm{H}$ & -0.05945 & 6.26595 & 1.45132 & $C$ & -6.82462 & 3.30405 & 1.67985 \\
\hline C & 3.02086 & 4.69033 & -0.61169 & $\mathrm{H}$ & -6.91389 & 3.92769 & 2.5647 \\
\hline $\mathrm{H}$ & 3.90907 & 5.23471 & -0.89987 & C & -7.20134 & -2.93083 & -1.0767 \\
\hline$C$ & -2.32918 & 5.66191 & -1.01562 & $\mathrm{H}$ & -7.44484 & -3.54129 & -1.9415 \\
\hline $\mathrm{H}$ & -2.63759 & 4.89547 & -1.72029 & $\mathrm{C}$ & -7.82555 & 3.31887 & 0.70698 \\
\hline$C$ & 6.28139 & -2.41613 & -0.88181 & $\mathrm{H}$ & -8.69414 & 3.95932 & 0.8286 \\
\hline $\mathrm{H}$ & 6.13454 & -1.85473 & -1.80033 & C & -3.17429 & -6.77133 & 1.18323 \\
\hline $\mathrm{C}$ & -1.92592 & -0.66407 & -0.04308 & $\mathrm{H}$ & -3.86894 & -6.89195 & 2.01024 \\
\hline C & 3.99402 & 2.29202 & -0.3787 & C & -8.06434 & -2.89105 & 0.01976 \\
\hline
\end{tabular}




\begin{tabular}{llllllll}
$\mathrm{C}$ & 5.38801 & -3.60198 & 1.02583 & $\mathrm{H}$ & -8.97939 & -3.47593 & 0.0145 \\
$\mathrm{H}$ & 4.54835 & -3.90859 & 1.64208 & $\mathrm{C}$ & 9.19464 & -3.85324 & 1.12359 \\
$\mathrm{C}$ & 7.75915 & 2.21182 & -0.17635 & $\mathrm{H}$ & 9.9012 & -3.82886 & 0.28893 \\
$\mathrm{H}$ & 8.58716 & 1.68015 & -0.63769 & $\mathrm{H}$ & 9.23761 & -4.84662 & 1.58121 \\
$\mathrm{C}$ & -2.729 & 2.80059 & 0.20033 & $\mathrm{H}$ & 9.54867 & -3.13586 & 1.87583 \\
$\mathrm{H}$ & -3.39333 & 3.63249 & 0.37755 & $\mathrm{C}$ & -7.74874 & -2.09375 & 1.12229 \\
$\mathrm{C}$ & 3.80547 & -2.51102 & -0.60503 & $\mathrm{H}$ & -8.41525 & -2.06094 & 1.9792 \\
$\mathrm{C}$ & -5.70364 & -1.36868 & 0.03279 & $\mathrm{C}$ & -3.71716 & -9.13688 & 0.45233 \\
$\mathrm{C}$ & 3.50516 & -1.19383 & -1.03205 & $\mathrm{H}$ & -3.86279 & -9.64358 & -0.50637 \\
$\mathrm{C}$ & -1.51113 & 7.67178 & 0.72869 & $\mathrm{H}$ & -3.14894 & -9.81889 & 1.09856 \\
$\mathrm{H}$ & -1.19929 & 8.44434 & 1.42639 & $\mathrm{H}$ & -4.69668 & -8.9904 & 0.91748 \\
$\mathrm{C}$ & 2.74552 & -3.45132 & -0.52416 & $\mathrm{C}$ & -3.24953 & 9.27317 & -0.18869 \\
$\mathrm{C}$ & 2.77419 & -4.85047 & -0.22862 & $\mathrm{H}$ & -3.15547 & 9.7952 & 0.76801 \\
$\mathrm{H}$ & 3.67457 & -5.43755 & -0.12466 & $\mathrm{H}$ & -2.79979 & 9.91504 & -0.95764 \\
$\mathrm{C}$ & -1.56126 & -5.39132 & -0.00404 & $\mathrm{H}$ & -4.31347 & 9.17978 & -0.42783 \\
$\mathrm{C}$ & -1.5285 & -2.89516 & -0.12235 & $\mathrm{H}$ & 1.38269 & 2.16899 & 0.42845 \\
$\mathrm{C}$ & -0.81258 & -4.1264 & -0.15674 & $\mathrm{H}$ & 1.06253 & -2.21128 & -0.88492 \\
$\mathrm{C}$ & 7.56836 & -2.75307 & -0.48235 & $\mathrm{H}$ & 8.41418 & -2.43573 & -1.08648 \\
\hline
\end{tabular}

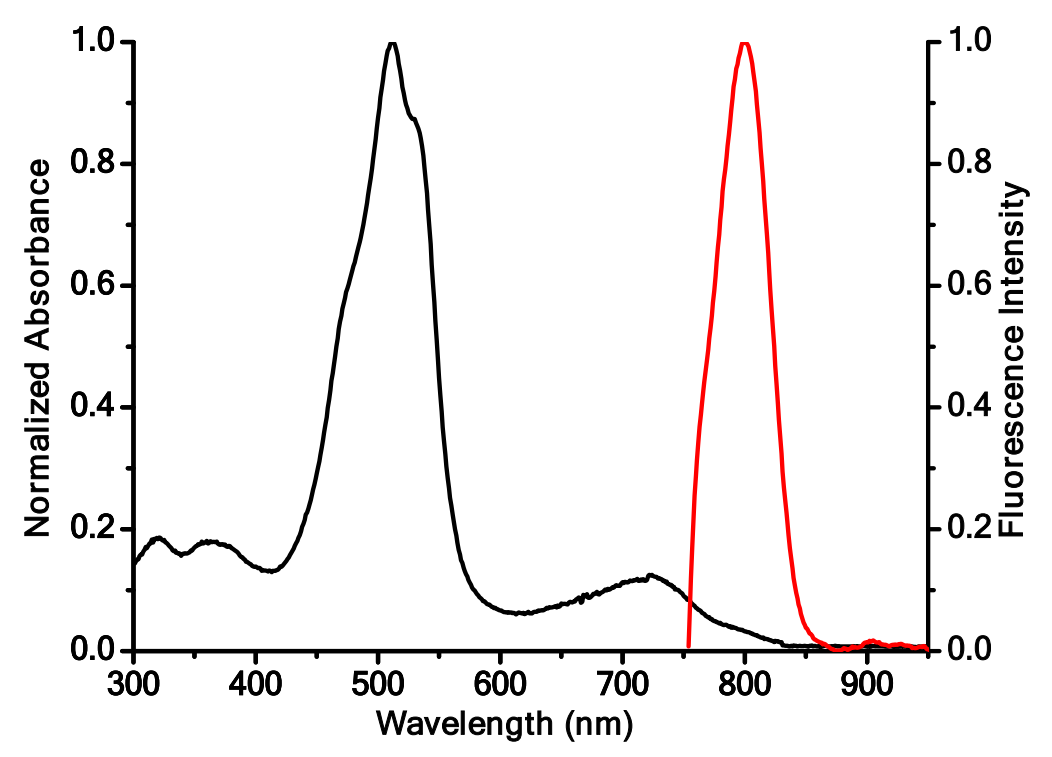

Figure S34: Normalized absorbance and fluorescence $\left(\lambda_{\mathrm{ex}}=510 \mathrm{~nm}\right)$ spectra of compound 3a in chloroform. 